\title{
Influence of aerosols, clouds, and sunglint on polarization spectra of Earthshine
}

\author{
Claudia Emde ${ }^{1}$, Robert Buras-Schnell ${ }^{2}$, Michael Sterzik ${ }^{3}$, and Stefano Bagnulo ${ }^{4}$ \\ ${ }^{1}$ Meteorological Institute, Ludwig-Maximilians-University, Theresienstr. 37, 80333 Munich, Germany \\ e-mail: claudia.emde@lmu.de \\ 2 Schnell Algorithms, Am Erdäpfelgarten 1, 82205 Gilching, Germany \\ 3 European Southern Observatory, Karl-Schwarzschild-Str. 2, 85748 Garching, Germany \\ ${ }^{4}$ Armagh Observatory \& Planetarium, College Hill, Armagh BT61 9DG, UK
}

Received 24 October 2016 / Accepted 23 April 2017

\begin{abstract}
Context. Ground-based observations of the Earthshine, i.e., the light scattered by Earth to the Moon, and then reflected back to Earth, simulate space observations of our planet and represent a powerful benchmark for the studies of Earth-like planets. Earthshine spectra are strongly linearly polarized, owing to scattering by molecules and small particles in the atmosphere of the Earth and surface reflection, and may allow us to measure global atmospheric and surface properties of planet Earth.

Aims. We aim to interpret already published spectropolarimetric observations of the Earthshine by comparing them with new radiative transfer model simulations including a fully realistic three-dimensional (3D) surface-atmosphere model for planet Earth.

Methods. We used the highly advanced Monte Carlo radiative transfer model MYSTIC to simulate polarized radiative transfer in the atmosphere of the Earth without approximations regarding the geometry, taking into account the polarization from surface reflection and multiple scattering by molecules, aerosol particles, cloud droplets, and ice crystals.

Results. We have shown that Earth spectropolarimetry is highly sensitive to all these input parameters, and we have presented simulations of a fully realistic Earth atmosphere-surface model including 3D cloud fields and two-dimensional (2D) surface property maps. Our modeling results show that scattering in high ice water clouds and reflection from the ocean surface are crucial to explain the continuum polarization at longer wavelengths as has been reported in Earthshine observations taken at the Very Large Telescope in 2011 (3.8\% and 6.6\% at $800 \mathrm{~nm}$, depending on which part of Earth was visible from the Moon at the time of the observations). We found that the relatively high degree of polarization of $6.6 \%$ can be attributed to light reflected by the ocean surface in the sunglint region. High ice-water clouds reduce the amount of absorption in the $\mathrm{O}_{2} \mathrm{~A}$ band and thus explain the weak $\mathrm{O}_{2} \mathrm{~A}$ band feature in the observations.
\end{abstract}

Key words. radiative transfer - polarization - scattering - techniques: polarimetric - techniques: spectroscopic - Earth

\section{Introduction}

More than 3500 exoplanets orbiting stars other than our Sun have been discovered so far and the quest to find life elsewhere in the universe has already started. The atmospheres of giant exoplanets are being scrutinized for their composition and in particular for possible thermochemical disequilibrium constituents (Stevenson et al. 2010). One of the greatest technical challenges is to overcome the enormous flux difference between an overwhelmingly bright star and the reflected light of a spatially unresolved planet. As a contrast enhancing technique, (spectro)polarimetry exploits the fact that the light of a solar type of star is unpolarized when integrated over the stellar disk, while it becomes polarized by molecular or particle scattering in the atmosphere of a planet or when it is reflected at its surface. Polarimetric signals provide a wealth of information about the atmosphere and surface of a planet. Molecular scattering produces the largest degree of polarization at a phase angle of $90^{\circ}$. Liquid droplets produce a particular phase angle dependence; for example, water clouds in the atmosphere of the Earth produce a large polarization feature at phase angles around $140^{\circ}$ corresponding to the rainbow. The polarized radiance spectrum includes additional information, for instance about trace gas concentrations of the atmosphere of a planet. Hence polarimetry is, in principle, an excellent tool for the detection and characterization of exoplanets (Seager et al. 2000; Schmidt et al. 2006).

Up to date, Earth is the only astronomical object that can be investigated in terms of bio-signatures unique to a life-hosting planet and can serve as the one and only benchmark for life as we know it. Observations of Earthshine allow us to observe these signatures through the reflected light of whole Earth from ground. Earthshine is the sunlight scattered by the dayside Earth and reflected back to Earth from the darker portion of the visible Moon. Different surface areas of the Earth can be probed as the relative Sun-Earth-Moon viewing geometry changes with the phase-angle.

Linear polarization spectra of Earthshine were obtained and interpreted by Sterzik et al. (2012) with the FORS2 instrument mounted at the ESO Very Large Telescope located in Chile. The useful spectral range covered a wavelength region between 450 and $920 \mathrm{~nm}$ with a resolution element of about $3 \mathrm{~nm}$. Two distinct viewing geometries of Earth were observed near quadrature (phase-angle near $90^{\circ}$ ). During the first observing epoch (dawn on April 25 2011), Earthshine contained contributions from the Atlantic sea, the Amazonas, and parts of Europe and Africa. The second observing epoch (dusk on June 92011 ) probed the 
Pacific side of Earth with almost no land surfaces visible. The interpretation of the results critically depends on detailed comparisons with theoretical models. Using the data of the vector radiative transfer models of Stam (2008), the fractional contribution of clouds and ocean surface can be inferred and small amounts of vegetated areas can be diagnosed.

But important features of the observed spectra remained unexplained. While the best models qualitatively match the blue parts of the spectrum (between 420 and $530 \mathrm{~nm}$ ) and allow us to derive cloud and ocean surface coverages similar to those resembled by satellite data, the red parts of the theoretical spectra are too low and do not fit the observations at all. The spectral slope of the observations is in general much flatter (and significantly higher in the red part, between 500 and $900 \mathrm{~nm}$ ) than predicted by the models available. For example, the spectropolarimetric observations by Sterzik et al. (2012) show a decreasing degree of polarization from around $10 \%$ to $5 \%$ in the spectral region from $500 \mathrm{~nm}$ to $920 \mathrm{~nm}$, while the predictions of the simulations by Stam (2008) are around $10 \%$ at $500 \mathrm{~nm}$, but less than $1 \%$ at $920 \mathrm{~nm}$. The finding of a relatively high polarization degree of Earthshine in the red spectral region has been corroborated by several other measurements, such as Bazzon et al. (2013), Takahashi et al. (2013), and Miles-Paéz et al. (2014). Although the observations and their analysis are challenging (e.g., applying a correct background subtraction of the Earthshine spectra), all observations point in the same direction and may rather hint to limitations in the model assumptions and their prescriptions.

Stam (2008) provide results of model calculations including polarization spectra of various model planets. The dataset was not created specifically to interpret the Earthshine observations by Sterzik et al. (2012), in particular the correct instrument filter function was not considered and ice clouds in high altitudes were not included. Thus it is not surprising that these model data do not fit the observations well and that we need a more realistic model setup. Stam (2008) obtain the Stokes vector of light scattered by planetary atmosphere as follows: the planet is treated as a scattering particle and its scattering phase matrix is computed for all phase angles simultaneously with series expansions. To calculate the elements of the planetary scattering matrix, these authors applied the algorithm by Stam et al. (2006) based on the accurate doubling-and-adding radiative transfer code by de Haan et al. (1987). This method works only for homogeneous planets, i.e., when the atmosphere is defined by $1 \mathrm{D}$ profiles and the surface is defined by one specific surface reflection function. To generate datasets with different mixtures of constituents, Stam (2008) approximate the light reflected by horizontally inhomogeneous planets via weighted sums of light reflected by horizontally homogeneous planets. In particular, Stam (2008) presents simulations for pure Rayleigh scattering atmosphere, Lambertian surfaces (including a spectral albedo), Fresnel reflecting surfaces, and liquid water clouds. The disk-integration method described in Stam et al. (2006) can also be applied to inhomogeneous planets. Karalidi \& Stam (2012) investigated the validity of the approximation of horizontally inhomogeneous planets by a weighted sum of homogeneous planets. These authors divided the planet into pixels that can have different reflection properties. For each pixel the Fourier components of the reflection matrix are computed and from those the disk-integrated signal is derived. They found that for the intensity and degree of polarization the impact of inhomogeneity is significant.

Simulations for a planet covered with liquid and ice water clouds have been presented by Karalidi et al. (2012). As Stam (2008) has already shown, the strong rainbow feature in the degree of polarization may be used to detect liquid water clouds on exoplanets. Ice clouds above liquid clouds dampen the rainbow feature, but according to the simulations by Karalidi et al. (2012) this feature should still be sufficiently strong to be detected in an exoplanet with Earth-like cloud cover. As the simulations by Karalidi et al. (2012) were performed only at three wavelengths $(550 \mathrm{~nm}, 660 \mathrm{~nm}$ and $865 \mathrm{~nm})$, the detailed spectral slope of the degree of polarization is unknown.

We developed a novel Monte Carlo approach that allows us to simulate the whole planet in full spherical geometry in one radiative transfer simulation, making a disk integration scheme redundant. This approach was combined with an importance sampling method that allows us to simulate the full spectrum based on photon paths calculated for one wavelength. We used a backward Monte Carlo approach as in García Muñoz \& Mills (2015) and García Muñoz (2015), however we used a different sampling method for the photon directions after scattering events. We included planet, star, and receiver in our simulation; the field of view of the receiver includes the full planet, which is illuminated according to the geometry (i.e., star and receiver positions with respect to planet). Thus we obtain the (spatially resolved) radiance measured by the receiver without any approximations related to the geometry; for example, we do not need to assume a locally plane-parallel atmosphere.

We validated our approach by comparison to the spectra provided by Stam (2008) and in general find a very good agreement for the same input assumptions. In this contribution we demonstrate with our method that in particular scattering in high ice water clouds and reflection at the ocean surface have a high impact on the expected degree of polarization and could explain the measurements. When we use a realistic Earth model, including three-dimensional (3D) distributions of cloud ice water and liquid water content and a two-dimensional (2D) surface albedo map, we obtain a good match with the observations.

The paper is organized as follows: Sect. 2 briefly describes our Monte Carlo code MYSTIC and our approach to simulate polarized radiances reflected from an illuminated planet. For validation we show spectral simulations for a molecular atmosphere and compare those to the data by Stam (2008). Section 3 shows observed polarimetric Earthshine spectra. Section 4 focuses on the degree of polarization in the $\mathrm{O}_{2} \mathrm{~A}$ band region. In Sect. 5 we evaluate the impact of typical Earth-like aerosols on the polarized radiance spectra, and we present a sensitivity study for liquid and ice clouds. In Sect. 6 we use our findings to interpret the observations by Sterzik et al. (2012) qualitatively. Finally, Sect. 7 presents a summary of our results and conclusions and provides an outlook.

\section{Monte Carlo approach to simulate polarization spectra of Earthshine}

\subsection{Vector radiative transfer model for Earth-like planetary atmospheres}

All simulations were performed via the radiative transfer model MYSTIC (Monte Carlo code for the phYsically correct Tracing of photons in Cloudy atmospheres; Mayer 2009), which is a versatile Monte Carlo code for atmospheric radiative transfer. This code is operated as one of several radiative transfer solvers of the libRadtran software package. The one-dimensional (1D) version of MYSTIC is freely available at www . libradtran . org (Emde et al. 2016) and the full 3D version used here is available for scientific use in joint projects. The MYSTIC code may be used to calculate polarized solar and thermal radiances and also 
Table 1. Model setups for simulations shown in Sects. 2-6.

\begin{tabular}{|c|c|c|c|c|c|c|c|}
\hline Section & $\begin{array}{l}\text { Model } \\
\text { geometry }\end{array}$ & Molecules & Aerosols & Clouds & Surface & ALIS & $\begin{array}{l}\text { Sample } \\
\text { resolution }\end{array}$ \\
\hline 2.2 & $3 \mathrm{D}^{1}$ & US-standard ${ }^{3}$ & - & 3D ECMWF 6 & 2D MODIS 7 & - & $100 \times 100$ \\
\hline 2.3 & $1 \mathrm{D}^{2}$ & McClatchey ${ }^{4}$ & - & - & $\begin{array}{l}\text { Lambertian } \\
\text { or ocean-BPDF }\end{array}$ & yes & $1 \times 1$ \\
\hline 4.1 & $1 D^{2}$ & MLS $^{3}$ & - & - & black surface & yes & $1 \times 1$ \\
\hline 5.1 & $1 D^{2}$ & MLS $^{3}$ & OPAC $^{5}$ & - & Lambertian & yes & $1 \times 1$ \\
\hline 5.2 & $1 D^{2}$ & MLS $^{3}$ & - & 1D layer & black surface & yes & $1 \times 1$ \\
\hline 5.2 & $1 \mathrm{D}^{2}$ & MLS $^{3}$ & - & 1D layer & Lambertian & yes & $1 \times 1$ \\
\hline 6.1 & $1 D^{2}$ & $\mathrm{MLS}^{3}$ & - & $\begin{array}{l}\text { 1D layer } \\
\text { or ocean-BPDF }\end{array}$ & Lambertian & yes & $1 \times 1$ \\
\hline 6.2 .1 & $3 \mathrm{D}^{1}$ & US-standard ${ }^{3}$ & - & 3D ECMWF 6 & 2D MODIS 7 & - & $500 \times 500$ \\
\hline $6.2 .1\left(\mathrm{O}_{2} \mathrm{~A}\right)$ & $3 \mathrm{D}^{1}$ & US-standard ${ }^{3}$ & - & 3D ECMWF 6 & 2D MODIS 7 & yes & $1 \times 1$ \\
\hline 6.2 .2 & $3 \mathrm{D}^{1}$ & US-standard ${ }^{3}$ & - & 3D ECMWF 6 & ocean-BPDF & - & $100 \times 100$ \\
\hline $6.2 .2\left(\mathrm{O}_{2} \mathrm{~A}\right)$ & $3 D^{1}$ & US-standard ${ }^{3}$ & - & 3D ECMWF 6 & ocean-BPDF & yes & $1 \times 1$ \\
\hline
\end{tabular}

Notes. ${ }^{(1)}$ Spherical planet with inhomogeneous atmosphere in three spatial dimensions; ${ }^{(2)}$ spherical planet, spherically symmetric atmosphere (horizontally homogeneous, vertically inhomogeneous); ${ }^{3)}$ molecular atmosphere with 51 vertical layers (Anderson et al. 1986); ${ }^{(4)}$ molecular atmosphere with 16 vertical layers, as defined in Stam (2008), Table 1; ${ }^{(5)}$ OPAC aerosol, standard 1D profiles from libRadtran (Emde et al. 2016), 13 vertical layers; ${ }^{(6)}$ liquid and ice water cloud fields from ECMWF model, $720 \times 360 \times 20$ grid cells covering the whole planet; ${ }^{(7)}$ global MODIS surface albedo data, $720 \times 360$ ground pixels, Lambertian.

to determine irradiances, actinic fluxes, and heating rates. The model has been applied extensively to generate realistic synthetic polarized satellite measurements for remote sensing of the Earth. These data have been used for the validation of various retrieval algorithms for cloud and aerosol optical and microphysical properties (e.g., Davis et al. 2013; Stap et al. 2016). The MYSTIC code allows the definition of arbitrarily complex $3 \mathrm{D}$ clouds and aerosols, an inhomogeneous surface albedo, and topography.

Polarized surface reflection is also included for ocean. It should be noted that it is not yet possible to combine bidirectional surface reflection functions (BPDFs) and Lambertian surface albedos into a 2D surface reflectance properties map. Further BPDFs for land surfaces are not included yet.

The model can be operated in fully spherical geometry. The implementation of 1D spherical geometry is described in Emde \& Mayer (2007). In order to simulate observations of inhomogeneous exoplanets as observed by a camera or telescope far away from the planet, we included 3D spherical geometry in MYSTIC following Deutschmann et al. (2011).

We implemented polarization with a combination of the following two methods (for details refer to Emde et al. 2010). First, the local estimate method (Marchuk et al. 1980; Marshak \& Davis 2005) was adapted to account for polarization, which is essential for accurate radiance simulations. Second, an importance sampling method was used to sample the photon direction after scattering or surface reflection.

We included sophisticated variance reduction methods (Buras \& Mayer 2011), which allowed us to calculate unbiased radiances for scattering media that are characterized by strongly peaked phase functions without approximations, such as delta scaling or truncation of the phase function. The variance reduction methods have been validated in a model intercomparison study including scattering media with strongly peaked phase functions (Kokhanovsky et al. 2010).

The MYSTIC code has been validated against benchmark data and in model intercomparison studies including discrete ordinate, doubling-and-adding, and Monte Carlo approaches to solve the radiative transfer problem. The implementation of polarization has been in particular validated in Kokhanovsky et al. (2010) and Emde et al. (2015), where results from MYSTIC agreed with the commonly established benchmark results within its standard deviation. Of particular relevance for the simulation of polarized spectra is the absorption lines importance sampling method (ALIS, Emde et al. 2011), which allows the calculation of full spectra by tracing photons at only one wavelength. The method can be applied to calculate broadband spectra in moderate resolution or small regions in very high spectral resolution. The method currently has one limitation, which is that when 3D cloud or aerosol fields are included, the optical properties of the clouds or aerosols are assumed to be spectrally constant. This assumption is fine for narrowband simulations, for example, the $\mathrm{O}_{2}$ - A band region, but not for the full spectrum from $400 \mathrm{~nm}$ to $1000 \mathrm{~nm}$.

The performance of our model for the simulation of polarized Earthshine spectra is summarized in Table 4. In the following sections different model setups are used, for example, 1D spherically symmetric atmospheres in combination with ALIS or 3D inhomogeneous atmospheres without using ALIS; these setups are summarized in Table 1. All simulations were performed using the MYSTIC solver included in libRadtran, version 2.0.1.

\subsection{Simulated pictures of the Earth}

In order to demonstrate our model setup, we simulated the image of Earth as recorded from space by a CCD camera. The image of the camera includes the full Earth and central point of the image is at $0^{\circ}$ latitude and $0^{\circ}$ longitude. We defined the position of the Sun by the point of intersection between the Earth surface and the connecting line between Earth center and Sun center. The intersection point is specified by latitude and longitude. The latitude of the sun position in this simulation is $0^{\circ}$, and in this case the longitude corresponds exactly to the phase angle. We simulated longitudes (i.e., phase angles) of $0^{\circ}, 30^{\circ}, 60^{\circ}$, and $90^{\circ}$. The model atmosphere includes molecules according to the US standard atmosphere by Anderson et al. (1986). We 
included a realistic 2D Lambertian surface albedo map derived from MODIS data (Schaaf et al. 2002). Further we included 3D cloud data (liquid and ice water clouds) from the European Centre for Medium-Range Weather Forecasts (ECMWF) model ${ }^{1}$. We used the operational $9 \mathrm{~h}$ forecast from 25 April 2011, 0 UTC. The data include 3D fields of liquid water content, ice water content, and the sub-grid cloud cover. The spatial resolution of the data is $0.5^{\circ}$ in latitude and $0.5^{\circ}$ in longitude. The vertical dimension in the ECMWF model is pressure, which we transfered to altitude using the hydrostatic equation. We interpolated the data on 20 altitude layers ( 0 to $20 \mathrm{~km}$ with $1 \mathrm{~km}$ resolution). Thus, in total we obtained $720 \times 360 \times 20$ grid cells including ice water content and liquid water content, respectively. Since a grid cell $\left(0.5^{\circ}\right.$ latitude times $0.5^{\circ}$ longitude) is more than $50 \times 50 \mathrm{~km}^{2}$ large at the equator, it is usually not completely cloud covered or completely cloud free. Thus, the ECMWF model provides a sub-grid cloud cover, which is a number between 0 and 1 and gives the fraction of the grid cell that is cloudy (1 means fully cloudy, 0 means cloud free). The cloud water content is given in grams of water per cubic meter of air and refers only to the cloudy part of the grid cell. We multiplied the ice water content and the liquid water content with the sub-grid cloud cover to obtain the correct water contents in each grid cell. For simplicity we used constant effective radii of $10 \mu \mathrm{m}$ (typical for liquid water cloud droplets) and $30 \mu \mathrm{m}$ (typical for ice crystals) as the ECMWF data do not include cloud particle sizes. Internally, in the ECMWF model parameterizations were used to calculate effective radii of cloud particles. We chose the wavelength of $550 \mathrm{~nm}$, where we expected to see the surface and also relatively strong polarization due to Rayleigh scattering. For this example we calculated an image with $100 \times 100$ pixels. We used the backward Monte Carlo tracing technique and calculate pixel by pixel sequentially. The initial photon direction was randomly determined within the field of view of the individual pixels. For each pixel we ran $10^{6}$ photons, resulting in $10^{10}$ photons for the full image.

In order to simulate the measured Earthshine spectra for a horizontally inhomogeneous planet as shown here, we do not need to calculate a spatially resolved image, but we may sample all photons reflected or scattered by the Earth into one large pixel. In the backward Monte Carlo method the initial photon directions are randomly distributed within a field of view including the full planet. In this case we needed much fewer photons to reach a good accuracy, i.e., $10^{5}$ photons are usually sufficient to obtain a standard deviation smaller than $1 \%$. Table 4 includes computational times and the corresponding accuracy for various setups, for example, a monochromatic simulation at $550 \mathrm{~nm}$ for a fully realistic Earth model including 3D cloud fields and a 2D surface albedo map takes $29 \mathrm{~s}$ on one CPU. The images are not calculated to compare with the observation, but to see whether the Earth model looks realistic (like an image taken by a camera on a satellite) and to better understand the results.

Figure 1 shows the results of the simulations for images with $100 \times 100$ pixels. The rows correspond to the simulated phase angles of $0^{\circ}, 30^{\circ}, 60^{\circ}$, and $90^{\circ}$. In each row we plot the images of the Stokes vector components $I, Q$ and $U$, normalized to incoming solar irradiance. The numbers above the images correspond to mean values of the Stokes components, $\bar{I}=\sum I_{i} / N$, $\bar{Q}=\sum Q_{i} / N$ and $\bar{U}=\sum U_{i} / N$, where the index $i$ denotes the pixel and $N=100^{2}$ corresponds to the number of pixels. When we perform the simulation for one pixel only, the result corresponds exactly to $\bar{I}, \bar{Q}, \bar{U}$ and we do not need to average. The

\footnotetext{
1 Integrated forecast system IFS, http://www. ecmwf.int
}

images in the last column show the degree of linear polarization, which is calculated as $P_{i}=\sqrt{Q_{i}^{2}+U_{i}^{2}} / I_{i}$. We need to calculate $P=\sqrt{\bar{Q}^{2}+\bar{U}^{2}} / \bar{I}$ to get the degree of polarization of the whole planet. This can be very different from the average of the individual values $P_{i}$.

For homogeneous planets $P$ is exactly 0 at a phase angle of $0^{\circ}$ for symmetry reasons. The patterns of $Q$ and $U$ are symmetric with positive and negative signs, i.e., positive and negative polarization values cancel each other when we observe the planet as a whole. The inhomogeneous surface and clouds break the symmetry, therefore the degree of polarization is nonzero. For our cloud and surface distribution, the degree of polarization is very small $(P=0.002)$. For individual pixels, $P_{i}$ can be as large as 0.1 . The degree of linear polarization $P$ increases with increasing phase angle. We would expect the maximum at $90^{\circ}$ for a pure molecular atmosphere over a black surface because here we have very strong polarization due to Rayleigh scattering. The images show that $P$ increases with phase angle and at $90^{\circ}$ phase angle we obtain $P=0.246$. The phase angle dependence clearly shows the influence of molecular scattering. The individual $P_{i}$ in clear-sky regions above ocean can be larger than 0.9 .

The Earth surface is clearly visible in the images of $I$ and $P$ but only weakly visible in $Q$ and $U$ because for the simulation we assumed that the surface is a Lambertian reflector, which by definition reflects unpolarized radiation. The polarization by scattering of photons that have been reflected by the surface is very small because the photon directions after surface reflection are random. The effects of the polarized surface reflection in the sunglint region over ocean are discussed later in Sect. 6.2.2. The maxima of $Q_{i}$ and $U_{i}$ are at the limb of the Earth due to second order of scattering.

We do not show the Stokes component $V$ corresponding to circular polarization because it is very small. Molecular scattering does not cause any circular polarization and the circular polarization by scattering at aerosols, cloud droplets, and ice crystals is several orders of magnitude smaller than linear polarization.

\subsection{Comparison to dataset by Stam (2008)}

To validate our approach we first calculated the same scenarios as Stam (2008) for the spectral range from 300 to $1000 \mathrm{~nm}$ for a phase angle of $90^{\circ}$. We used the ALIS method to simulate the spectrum. Our model yields the radiance averaged over the full quadratic field of view, rather than the average over the planetary disk only. Therefore, to obtain the same normalization as Stam (2008), we need the following conversion factor:

$N=\pi \frac{(2 d \tan \alpha)^{2}}{\pi r_{\mathrm{E}}^{2}} \approx \frac{4(\alpha d)^{2}}{r_{\mathrm{E}}^{2}}$.

Here $\alpha$ is the angle defining the quadratic field of view of the simulation, $d$ is the distance between the observer and the center of the planet, in our case the distance between Earth and Moon, and hence $(2 d \tan \alpha)^{2}$ is the cross-sectional area of the field of view at the location of the Earth. The area within the field of view covered by the planet is $\pi r_{\mathrm{E}}^{2}$, where $r_{\mathrm{E}}$ is the radius of the Earth. The additional factor $\pi$ is required for consistency with the Stokes vector definition by Stam (2008).

Figure 2 shows MYSTIC simulations in comparison to data by Stam (2008). We ran $10^{6}$ photons and the relative standard deviation is generally below $1 \%$ for all Stokes parameters and 

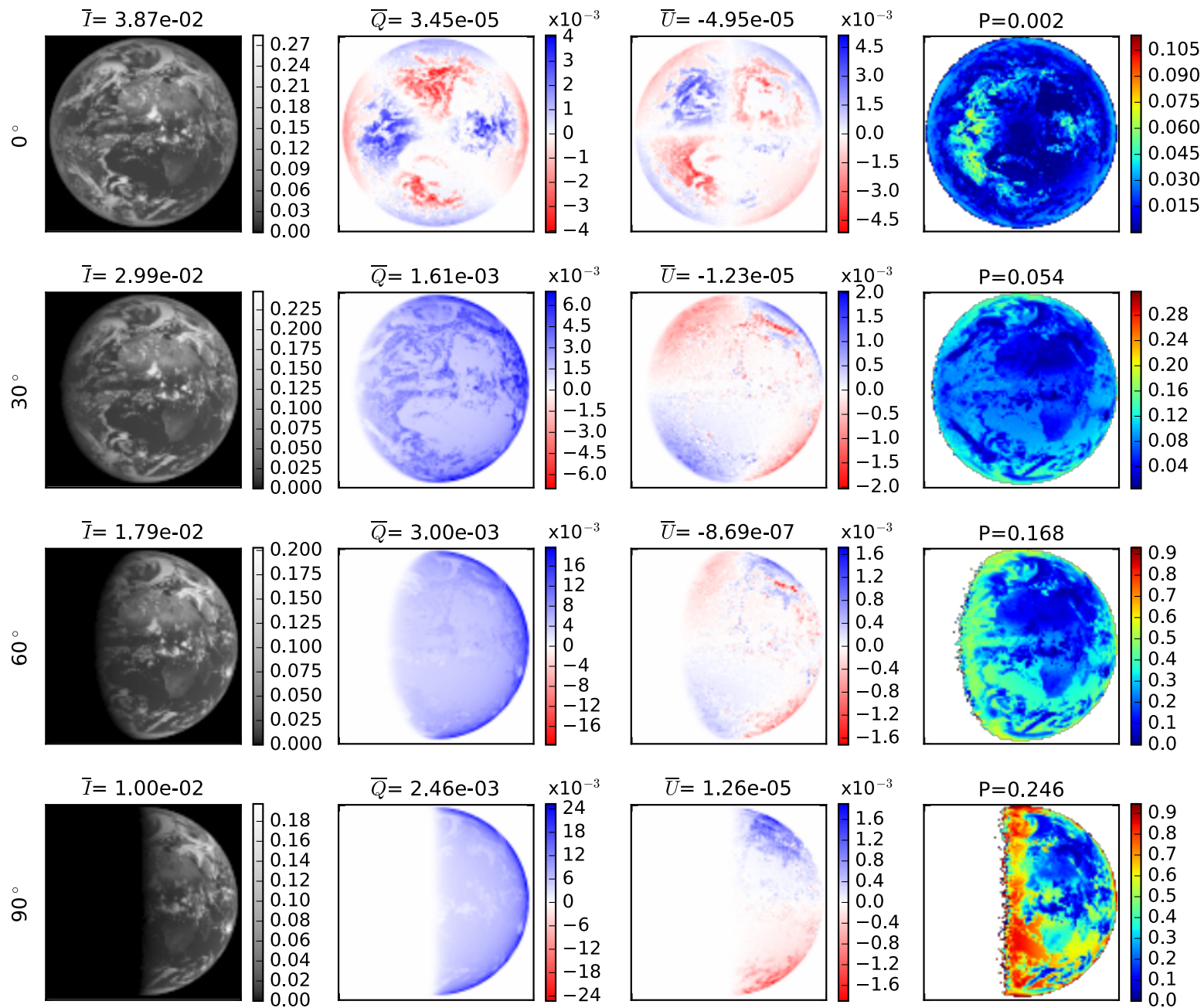

Fig. 1. Example simulation including a Lambertian surface albedo map and 3D cloud data from the ECMWF weather model. The rows correspond to phase angles of $0^{\circ}, 30^{\circ}, 60^{\circ}$, and $90^{\circ}$. The columns show the Stokes vector components $I, Q$, and $U$ and the degree of linear polarization. On top of each Stokes component figures the mean values of all pixels in the image are given, and the right panels include polarization calculated as $P=\sqrt{\bar{Q}^{2}+\bar{U}^{2}} / \bar{I}$.

also for the degree of polarization. The bottom panels show the absolute differences between MYSTIC and the data by Stam.

We generally find very good agreement for Lambertian surfaces. Here we tried to adapt the scenario by Stam (2008) as close as possible. We used the standard atmosphere by McClatchey et al. (1972) and added oxygen with a concentration of $21 \%$. For absorption Stam (2008) has used a $k$ distribution (Stam et al. 2000), whereas we used the REPTRAN parameterization by Gasteiger et al. (2014) in coarse resolution $\left(15 \mathrm{~cm}^{-1}\right)$. These different approaches explain the differences in the absorption bands.

For the land surface we used the spectral albedo of grass as measured by Feister \& Grewe (1995). These measurements are available for the spectral region from $290 \mathrm{~nm}$ to $800 \mathrm{~nm}$. Above $800 \mathrm{~nm}$ we used a constant albedo of 0.587 , corresponding to the measurement at $800 \mathrm{~nm}$. Stam (2008) used data for deciduous forest from the ASTER spectral library. The spectral albedos are similar, in particular they both show a local maximum between $500 \mathrm{~nm}$ and $600 \mathrm{~nm}$ (due to absorption bands of chlorophyll) and both show a high albedo at wavelengths longer than $700 \mathrm{~nm}$. The difference seen in Fig. 2 for land surfaces (green lines) are due to the different spectral albedo data.

Stam (2008) treats the ocean as a Fresnel surface, i.e., a flat surface neglecting the influence of oceanic waves. For the
MYSTIC simulations we used a reflection matrix that is also based on the Fresnel equations and additionally takes into account the influence of the waves including shadowing effects (Mishchenko \& Travis 1997; Cox \& Munk 1954a,b; Tsang et al. 1985). We chose a small value of $1 \mathrm{~m} / \mathrm{s}$, which produces a narrow glint and should compare better with the pure Fresnel surface than more realistic larger wind speeds. Our model produces larger $I$ and $Q$ values than the pure Fresnel surface; also the degree of polarization is slightly larger.

\section{Polarimetric observations of the Earthshine}

Figure 3 shows observed data of the FORS instrument (blue line). The Earthshine is sunlight scattered by the dayside Earth, which is reflected by the nightside of the Moon. We therefore needed to multiply the modeled Stokes vector with the lunar depolarization matrix to compare our model results with the Earthshine observation. Unfortunately this matrix is not known yet, and this is indeed the largest uncertainty for the interpretation of Earthshine measurements. We multiplied the observed data with a lunar depolarization factor $\delta$ to get a rough estimate of the degree of polarization of the Earthshine, without reflection at the lunar surface. 

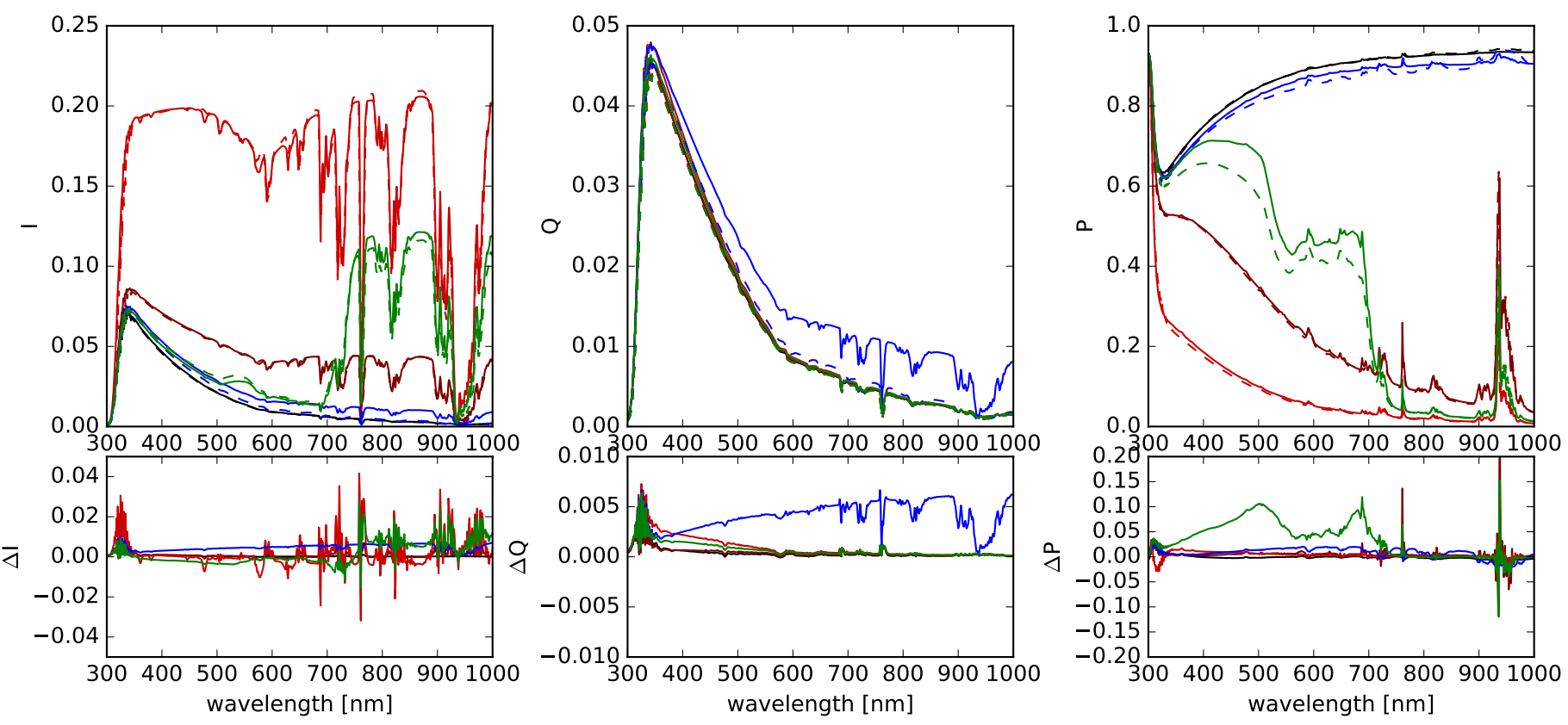

\begin{tabular}{|c|c|c|c|c|c|c|c|c|c|}
\hline & & & & & urface type: & & & & \\
\hline - & Lambert 0.0 & - & Lambert 0.2 & - & Lambert 1.0 & - & Ocean / Fresnel & - & Gras / Forest \\
\hline
\end{tabular}

Fig. 2. Intensity $I$, Stokes parameter $Q$, and degree of polarization $P=Q / I$ (top). The solid lines show MYSTIC calculations and the dashed lines show results by Stam (2008). Lambertian surfaces with albedos 0, 0.2, and 1.0 are compared. For ocean and land surfaces (forest and grass) the surface properties are not exactly the same (see text for details). The small bottom plots show absolute differences between MYSTIC results and Stam (2008).

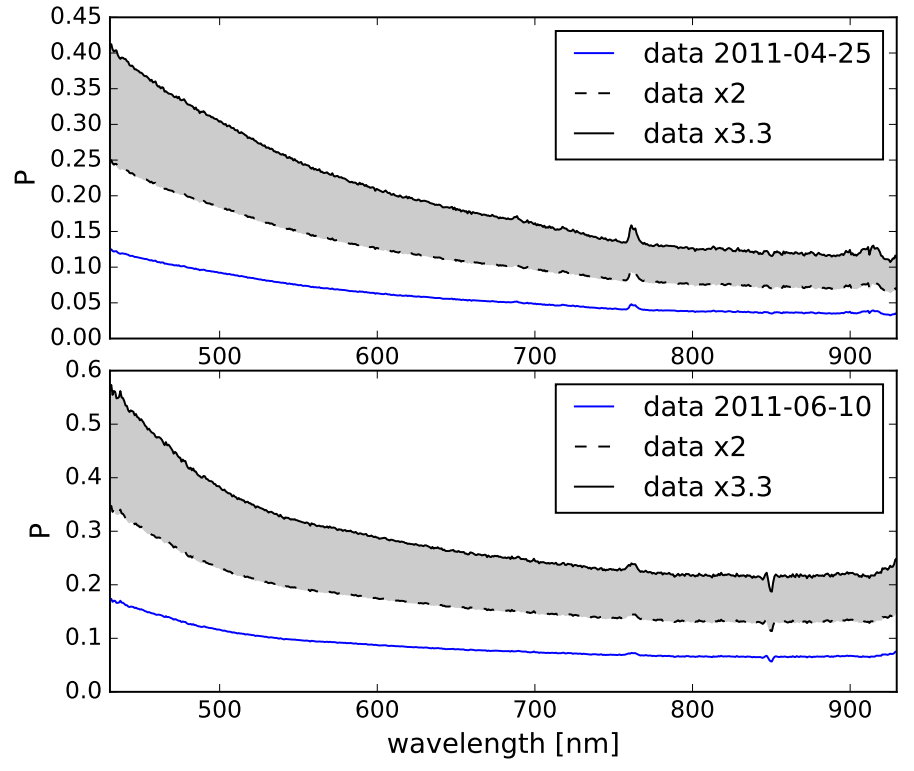

Fig. 3. Observations by FORS of the degree of polarization (blue line). The black lines are for different assumptions for moon depolarization; factor 3 (solid line) corresponds to Dolfuss (1957) and factor 2 (dashed line) corresponds approximately to Bazzon (2013, for lunar surface albedo 0.1).

Dollfus (1957) derived a constant value of $\delta=3.3$, whereas Sterzik et al. (2012) assumed $\delta=3.3 \cdot \frac{\lambda}{550}$, where $\lambda$ is the wavelength in $\mathrm{nm}$, thus increasing depolarization with wavelength. Bazzon et al. (2013) derived a polarization efficiency function $\epsilon(\lambda, a)(\epsilon=1 / \delta)$ based on polarized reflection and albedo measurements of several Apollo lunar soil samples (Hapke et al. 1993). The polarization efficiency function $\epsilon(\lambda, a)$ depends on surface albedo $a$ and on $\lambda$. For low albedos of 0.1 the polarization efficiency ranges from 0.57 at $450 \mathrm{~nm}$ to 0.48 at $800 \mathrm{~nm}$. For high albedos of 0.2 this value ranges from 0.37 at $450 \mathrm{~nm}$ to 0.21 at $800 \mathrm{~nm}$; these values correspond roughly to the assumption by Sterzik et al. (2012).

For the interpretation of the Earthshine observations the lunar depolarization factor is the largest uncertainty. In Fig. 3 the black lines show the measurement multiplied with $\delta=3.3$ (solid line) and multiplied with $\delta=2$ (dashed line), respectively. The gray area shows the possible range of the degree of polarization of the light scattered by the illuminated Earth. This corresponds to the model output, i.e., to an Earth observation of an imaginary instrument on the lunar surface.

\section{Degree of polarization in $\mathrm{O}_{2} \mathrm{~A}$-band region}

For the interpretation of spectral features such as the $\mathrm{O}_{2} \mathrm{~A}$ absorption band it is very important to perform the radiative transfer simulations at the same spectral resolution as the measurements (see also Boesche et al. 2008). The FORS observations were obtained with grism $300 \mathrm{~V}$ and a $2^{\prime \prime}$ slit width, yielding a spectral resolution of about 250 or $3 \mathrm{~nm}$ around the $\mathrm{O}_{2} \mathrm{~A}$ band.

In order to demonstrate the importance of spectral resolution we took a closer look at the $\mathrm{O}_{2} \mathrm{~A}$ band. The gray lines in the top plots of Fig. 4 show a simulation performed at a spectral resolution of $0.01 \mathrm{~nm}$. The absorption coefficients were obtained using the Atmospheric Radiative Transfer Simulator (ARTS ) line-byline model (Eriksson et al. 2011). For this calculation the standard midlatitude-summer atmosphere by Anderson et al. (1986) was used. The phase angle is $90^{\circ}$.

The Stokes vector calculated in high spectral resolution is convolved with the instrument filter function (red line in Fig. 4) to obtain the correct result. The bottom plot shows the same spectral region for different spectral resolutions calculated via 
accurate simulation with instrument filter function (FWHM 3nm)
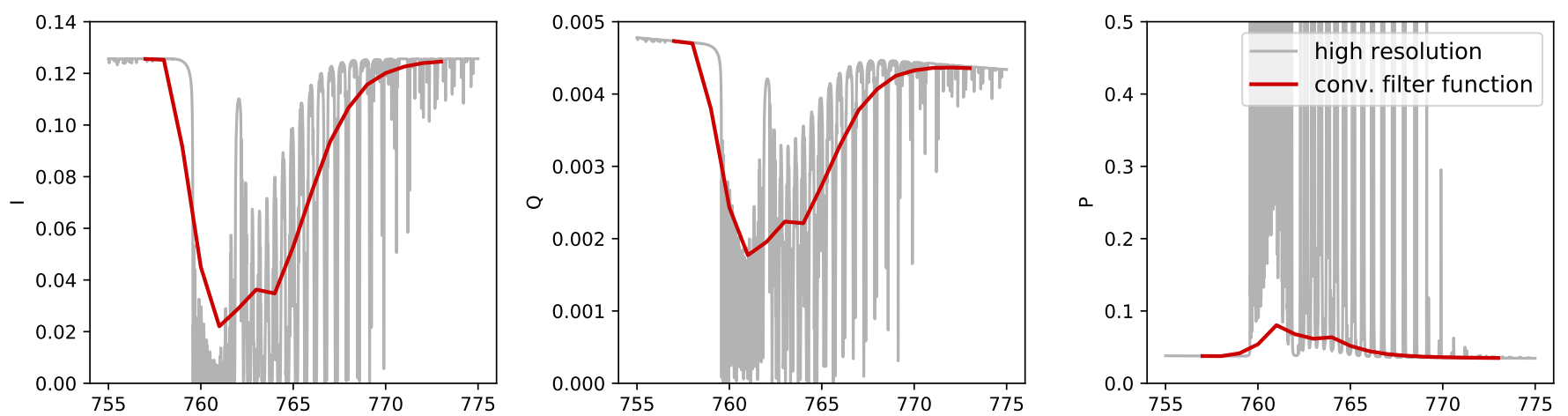

absorption parameterization with different spectral resolutions
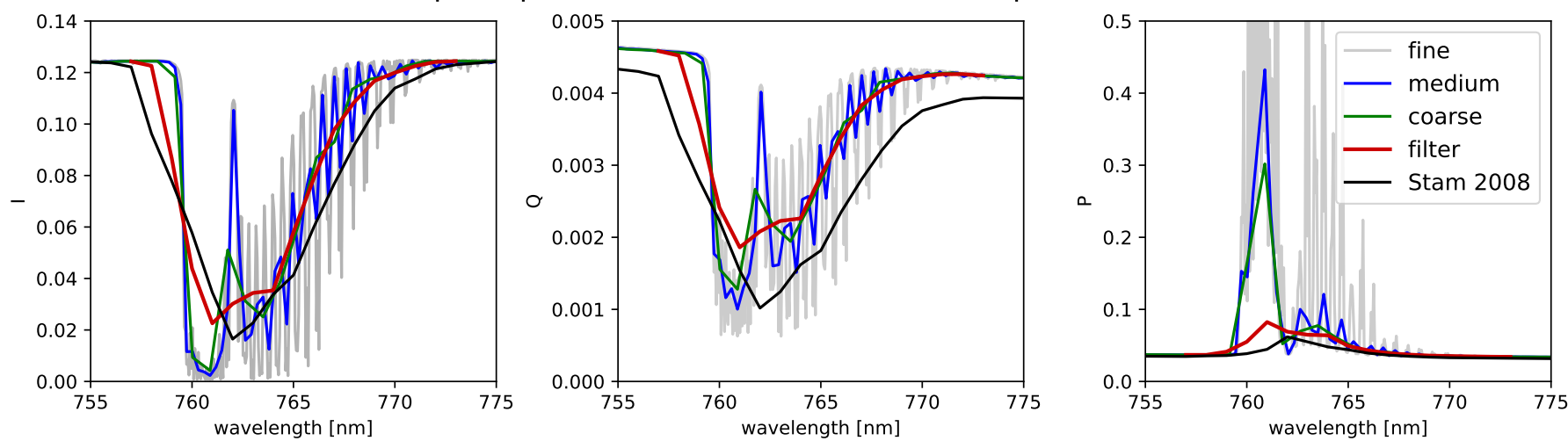

Fig. 4. Intensity $I$, Stokes parameter $Q$, and degree of polarization $P=Q / I$ in the $\mathrm{O}_{2}$ A band region. The top plots show high spectral resolution calculations with $\Delta \lambda=0.01 \mathrm{~nm}$ and the spectrum convolved with the instrument filter function. The bottom plots show the same spectral region calculated using the REPTRAN parameterization in three spectral resolutions (fine, medium, and coarse). The red line shows the REPTRAN calculation in coarse resolution convolved with the instrument filter function. For comparison, the data from Stam (2008) are also shown. The Lambertian surface albedo is 0.6 .

absorption parameterizations. For REPTRAN three resolutions are available: fine $\left(1 \mathrm{~cm}^{-1}\right.$ corresponding to $\approx 0.05 \mathrm{~nm}$ at $760 \mathrm{~nm})$, medium $\left(5 \mathrm{~cm}^{-1}\right.$ corresponding to $\left.\approx 0.3 \mathrm{~nm}\right)$, and coarse $\left(15 \mathrm{~cm}^{-1}\right.$ corresponding to $\left.\approx 1 \mathrm{~nm}\right)$. The red line in the bottom plots shows the REPTRAN simulation in coarse resolution convolved with the instrument filter function. The result is very close to the accurate simulation based on the simulation in high spectral resolution. Results shown throughout the paper are simulated using REPTRAN in coarse resolution and the resulting spectra are convolved with the FORS instrument filter function.

The data by Stam (Stam 2008, black line in bottom plots) are available on a $1 \mathrm{~nm}$ grid but their $k$-distribution method obviously averages over a wider wavelength range and does not include the FORS instrument filter function. The maximum degree of polarization depends to a large extent on the spectral resolution; this value decreases with coarser resolution due to averaging, therefore it is crucial to take into account the correct instrument filter function, in particular when spectral features are compared.

\section{Simulation of polarized Earthshine spectra for various atmospheric components}

To study the effect of various atmospheric constituents on the degree of polarization, we performed simulations separately for aerosols, liquid water clouds, and ice water clouds. The results of the sensitivity studies are presented in this section.

A phase angle of $90^{\circ}$ is a good observation geometry that yields high polarimetric signals for molecular atmospheres such as the atmosphere of the Earth, thus all simulations shown here are for this planet-sun-observer geometry. Further we simulated the full Earth as one pixel, which corresponds to a measurement of the Earthshine.

\subsection{Aerosols}

Figure 5 shows simulations for various standard aerosol mixtures (desert, continental average, and maritime clean), which are defined according to the Optical Properties of Aerosols and Clouds (OPAC) database (Emde et al. 2016; Hess et al. 1998). For all simulations in this and the following sections we included the midlatitude-summer atmosphere by Anderson et al. (1986). The top row represents a completely green planet. The polarization spectra clearly show the vegetation step at $700 \mathrm{~nm}$; this is the expected result because typical aerosol profiles have relatively small optical thicknesses of smaller than 0.5 and the surface is well visible. Compared to the clear-sky simulation the degree of polarization is lower at shorter wavelengths and higher at longer wavelengths. The reason is that at shorter wavelengths, Rayleigh scattering is much stronger and aerosol scattering causes smaller polarization than Rayleigh. At longer wavelengths, Rayleigh scattering is weak and the aerosols mask the depolarizing surface. Hence the degree of polarization becomes larger in the presence of aerosols. Above $750 \mathrm{~nm}$ the measured degree of polarization in the continuum corresponds approximately to the simulation with continental aerosol profile, but spectral absorption features are much stronger in the simulation than in the observation. 

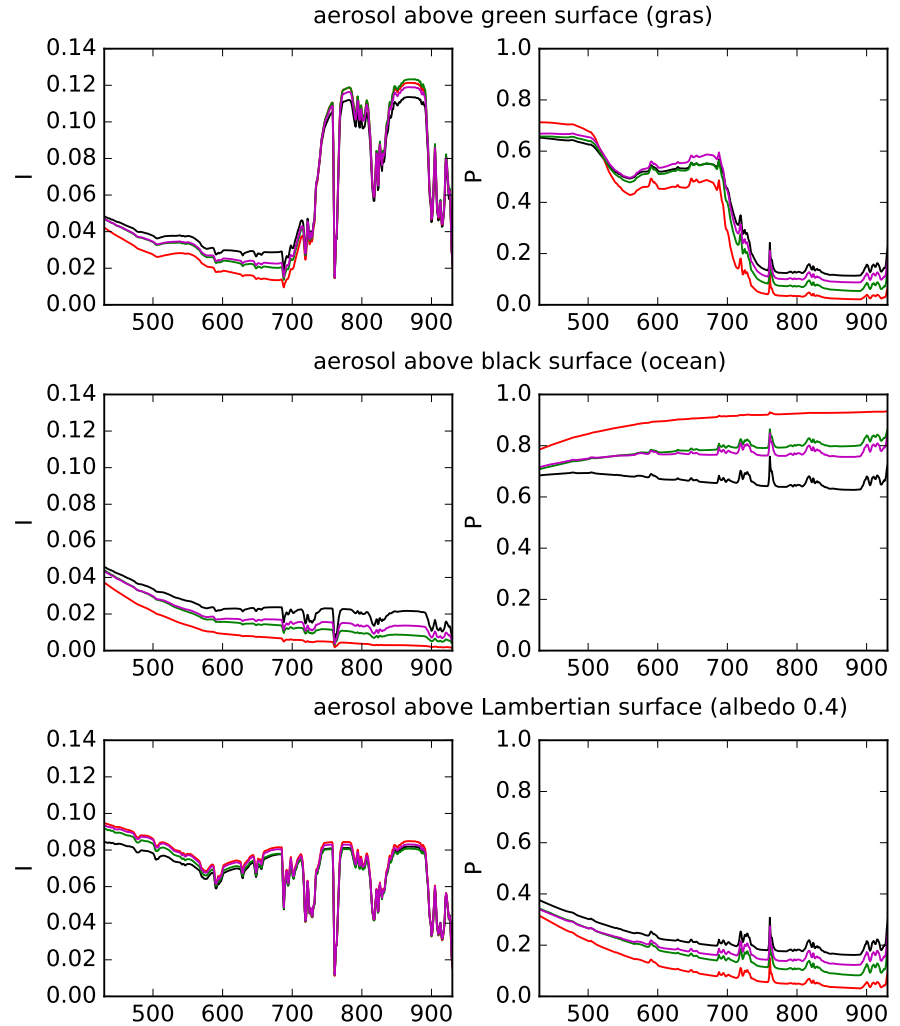

quasi inhomogeneous planet ( $25 \%$ gras, $50 \%$ ocean, $25 \%$ Lambert 0.4 )
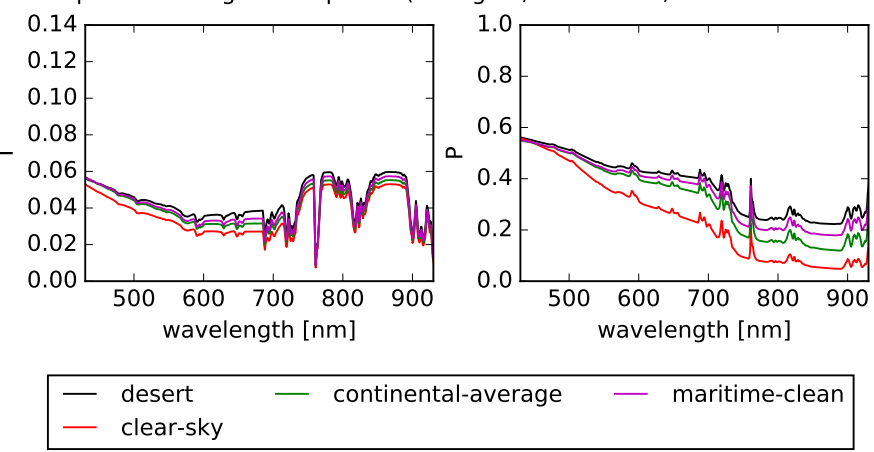

Fig. 5. Intensity $I$ (left panels) and degree of polarization $P$ (right pan$e l s$ ) for an atmosphere characterized by various aerosol mixtures above different surface types. From top to bottom: grass, ocean, Lambertian surface with albedo 0.4 , and mixed surface properties are shown.

The second row shows a simulation for a dark surface. Compared to clear sky, aerosol scattering decreases the degree of polarization by $10-20 \%$.

The third row shows a simulation for a Lambertian surface with an albedo of 0.4 , which is similar to a sand surface. Here, the degree of polarization is enhanced by aerosol scattering.

The fourth row shows a simulation for a "quasi" inhomogeneous planet. The various simulations were approximated by a weighted sum as in Stam (2008),

$\boldsymbol{I}(\lambda, \alpha)=\sum_{n=1}^{N} f_{n} \boldsymbol{I}_{n}(\lambda, \alpha) \quad$ with $\quad \sum_{n=1}^{N} f_{n}=1$,

where $N$ is the number of simulated horizontally homogeneous planets and $f_{n}$ are the respective fractions. Although the weighted sum approach yields significantly different results compared to simulations for inhomogeneous planets, it can be used to estimate roughly the influence of the various components on polarized radiance spectra.
We assumed the following fractions for the surface type: $25 \%$ grass, $50 \%$ ocean, and $25 \%$ Lambertian with an albedo of 0.4 . Here also, the degree of polarization is increased by aerosol scattering. Generally the smallest increase is observed by the continental-average aerosol mixture, followed by the maritimeclean aerosol. The desert aerosol mixture shows the largest impact. The results are dominated by the land surfaces because the total intensity reflected by the ocean is relatively small.

The results show that aerosols that are typically found in the atmosphere of the Earth cannot explain the observed polarization spectra. For the simulation with surface albedo 0.4 the degree of polarization is similar to the observations (see Fig. 3), but the spectral slope is different and, even more important, the spectral features are much weaker in the observations than in the simulation.

\subsection{Water clouds}

Figure 6 shows the sensitivity of the polarized radiance spectra on various water cloud parameters, i.e., cloud optical thickness, cloud altitude, and effective radius of cloud droplets. The cloud optical properties for the simulations were calculated using the Mie tool of the libRadtran package (Emde et al. 2016; Wiscombe 1980). Optical properties of single spherical droplets were averaged over a gamma size distribution with a constant effective variance of 0.1 and different effective radii. The surface albedo is 0 and aerosols are not included in the simulations. The impact of aerosols and surface on radiance simulations with water clouds is relatively small because the clouds hide the boundary layer including most aerosols and the surface.

The top plots show the sensitivity to cloud optical thickness at $550 \mathrm{~nm}$, which varies between typical values from 5 to 20 . The cloud layer is situated between 2 and $3 \mathrm{~km}$ altitude and the effective radius of the cloud droplets is $10 \mu \mathrm{m}$. The intensity $I$ increases with increasing cloud optical thickness because the thicker the cloud the more it reflects to space. The degree of polarization is decreased as the optical thickness increases, mainly because the highly polarizing Rayleigh scatterings are replaced by cloud scattering which polarize less. Also enhanced multiple scattering decreases the degree of polarization.

The middle plots show the sensitivity to cloud altitude. The cloud layer has an optical thickness of 10 and an effective droplet radius of $10 \mu \mathrm{m}$. The geometrical thickness of the cloud is always $1 \mathrm{~km}$ and the bottom height is varied from 2 to $8 \mathrm{~km}$. The intensity $I$ is almost invariant to cloud altitude because the amount of radiation reflected by the cloud depends mostly on optical thickness. The degree of polarization $P$ decreases with increasing cloud altitude because the cloud "hides" the molecular atmosphere below which the amount of Rayleigh scattering, which is mainly responsible for the polarization signal, decreases.

The bottom plots show the sensitivity to the effective radius, which is varied from 5 to $15 \mu \mathrm{m}$ for a cloud layer between 2 and $3 \mathrm{~km}$ with an optical thickness of 10 . We see that the intensity and degree of polarization are not very sensitive to cloud droplet size in this spectral region. The reason is that in the geometrical optics limit, which we may assume approximately for the given parameters, extinction is 2 for all droplet sizes and the imaginary part of the refractive index of liquid water is approximately 0 , i.e., extinction is only caused by scattering. The scattering phase matrix is also invariant to particle size in geometrical optics.

Overall we see that the degree of polarization in the red part of the spectrum is lower $(<5 \%$ for $\tau \geq 10)$ than in the observations (10-30\%). The observed slope of the polarization 
C. Emde et al.: Influence of aerosols, clouds, and sunglint on polarization spectra of Earthshine
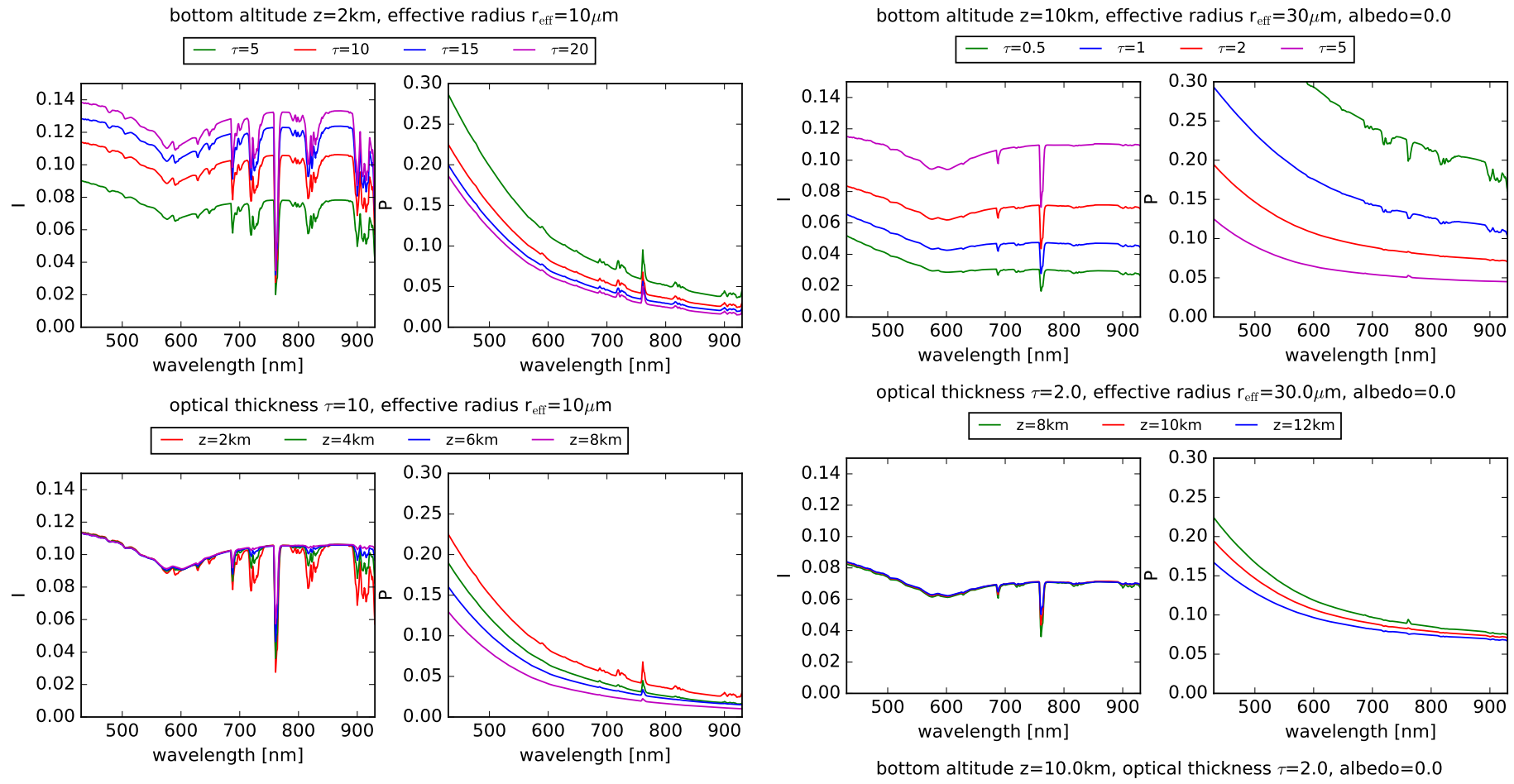

bottom altitude $z=2 \mathrm{~km}$, optical thickness $\tau=10$
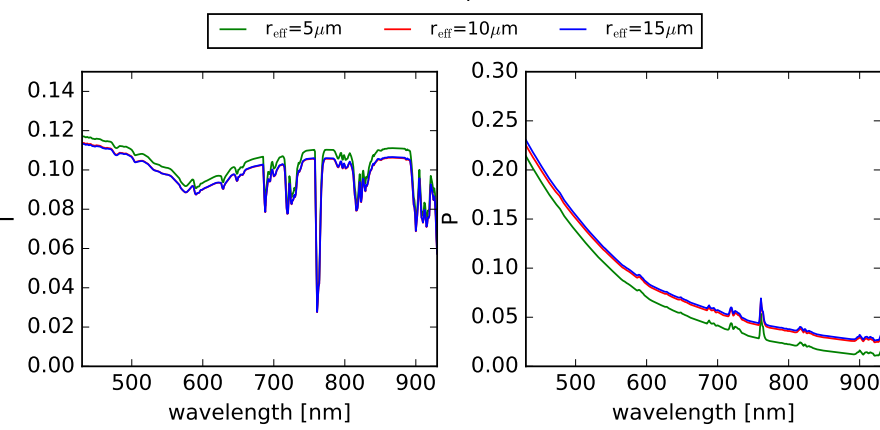

Fig. 6. Intensity $I$ and degree of polarization $P$. The top figures show the sensitivity to cloud optical thickness for a cloud layer from $2-3 \mathrm{~km}$ altitude including cloud droplets with an effective radius $r_{\text {eff }}$ of $10 \mu \mathrm{m}$. Cloud optical thicknesses $\tau$ at $550 \mathrm{~nm}$ range from 5 to 20 . The middle row shows the sensitivity to cloud altitude for a cloud with $\tau=10$ and $r_{\text {eff }}=10 \mu \mathrm{m}$. The bottom altitude of the $1 \mathrm{~km}$ thick cloud layer varies from 2 to $8 \mathrm{~km}$. The bottom figures show the sensitivity to effective radius for a cloud layer with a bottom altitude of $2 \mathrm{~km}$ and $\tau=10$. The effective radius varies from 5 to $15 \mu \mathrm{m}$.

spectrum $P$ (gray lines) is also flatter than all simulations including liquid clouds. All results are only valid for a phase angle of $90^{\circ}$, depending on phase angle the degree of polarization can be increased or decreased compared to pure molecular scattering. We have not investigated phase angles other than $90^{\circ}$ since the observations were performed close to $90^{\circ}$.

\subsection{Ice water clouds}

To study the sensitivity to ice water cloud parameters, we varied the ice cloud optical thickness at $550 \mathrm{~nm}$ between 0.5 and 5 , the cloud bottom altitude between $8 \mathrm{~km}$ and $12 \mathrm{~km}$, and the effective radius between $10 \mu \mathrm{m}$ and $60 \mu \mathrm{m}$. These values are typical for cirrus clouds on Earth; for example, Wang et al. (2011) have shown that ice optical thickness derived from MODIS data is in the range from about 0.1 and 6 . The effective diameter of the ice crystals is in the range from 20 to $120 \mu \mathrm{m}$. The mean values of
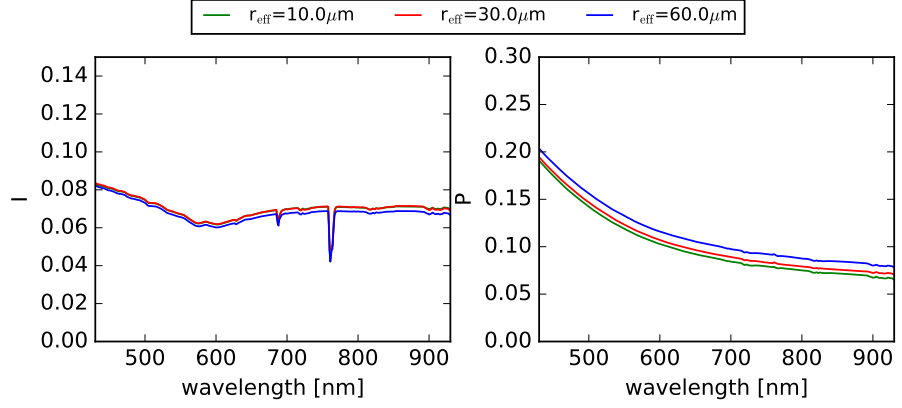

bottom altitude $z=10.0 \mathrm{~km}$, optical thickness $\tau=2.0, \mathrm{r}_{\text {eff }}=60 \mu \mathrm{m}$

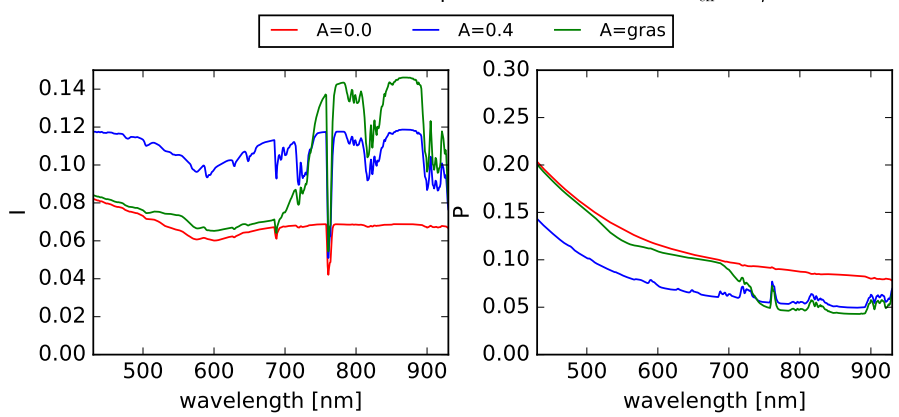

Fig. 7. Intensity $I$ and degree of polarization $P$. The top figures show the sensitivity to ice water cloud optical thickness (at $550 \mathrm{~nm}$ ) for a cloud layer from $10-11 \mathrm{~km}$ altitude including ice crystals with an effective radius $r_{\text {eff }}$ of $30 \mu \mathrm{m}$. Cloud optical thicknesses $\tau$ range from 0.5 to 5 . The second row shows the sensitivity to cloud altitude for a cloud with $\tau=2$ and $r_{\text {eff }}=30 \mu \mathrm{m}$. The bottom altitude of the $1 \mathrm{~km}$ thick cloud layer varies from 8 to $12 \mathrm{~km}$. The third row shows the sensitivity to the effective radius for a cloud layer with a bottom altitude of $10 \mathrm{~km}$ and $\tau=2$. The effective radius is varied from 10 to $60 \mu \mathrm{m}$. The bottom panels show the sensitivity to surface albedo.

the MODIS retrieval are 2.83 for optical thickness and 58.5 for effective diameter.

Figure 7 shows the intensity $I$ and the degree of polarization $P$ for a planet covered with a $1 \mathrm{~km}$ thick ice cloud layer. The ice cloud consists of ice crystals of various habits, such as solid 
columns, aggregates, and bullet rosettes. For the ice cloud optical properties the parameterizations for a general habit mixture by Heymsfield et al. (2013), Yang et al. (2013), and Baum et al. (2014) have been used. This mixture is composed of nine crystal shapes: solid/hollow bullet rosettes, solid/hollow columns, plates, droxtals, small/large aggregate of plates, and an aggregate of solid columns.

The top figures show the sensitivity to ice cloud optical thickness. The bottom altitude of the cloud is at $10 \mathrm{~km}$ and the surface albedo is 0 . As for the water cloud the intensity increases with increasing optical thickness and the degree of polarization decreases. Further we see that the spectral slope of $P$ decreases with increasing optical thickness. The slope is much flatter than for water clouds because the ice cloud layer is at a higher altitude and "hides" the Rayleigh scattering in the lower atmosphere.

For very small optical thicknesses of 0.5 and 1 we see that the degree of polarization becomes smaller in the absorption bands than in the continuum. A possible explanation is the following: For low optical thickness the path length in the cloud layer is small compared to the path lengths in the free molecular atmosphere. Therefore absorption reduces the number of strongly polarizing Rayleigh scattering events more than the number of much lower polarizing cloud scattering events.

Now we investigate how other cloud parameters influence $I$ and $P$. The second row in Fig. 7 shows the effect of cloud altitude; for the water cloud $I$ is almost insensitive to cloud height whereas both $P$ and its slope decrease with increasing cloud height. Here we also see that the strength of the $\mathrm{O}_{2} \mathrm{~A}$ absorption band depends on cloud altitude. The average length of photon propagation paths through the atmosphere is shorter for higher clouds; typically photons enter the atmosphere at the top, are scattered in the cloud, and leave the atmosphere. Therefore there is more molecular absorption when the cloud layer is at a lower altitude, which can be clearly seen in $I$. For $P$ the amplitude of the $\mathrm{O}_{2} \mathrm{~A}$ band decreases with increasing cloud height and for very high clouds $P$ becomes smaller in the $\mathrm{O}_{2} \mathrm{~A}$ band than in the continuum.

The third row shows the impact of effective radius: $I$ is almost invariant and $P$ slightly increases with increasing particle size. The slope of $P$ slightly decreases with increasing particle size.

The optical thickness of water clouds is usually larger than 10 so that the surface is not visible through the clouds. Ice clouds are optically thinner; for cirrus clouds the optical thickness is typically about 5 or even smaller and the surface can often be seen through the clouds. The bottom panels of Fig. 7 show simulations with different surfaces: for Lambertian surfaces with albedos 0.0 and 0.4 and for a green surface. In the simulation with the black surface the water vapor bands are not visible. Interestingly these become visible when there is surface reflection. Reflection by the surface increases the probability that photons that are transmitted by the cloud toward the surface are reflected back to space. Some of these photons are absorbed in the lower atmosphere so that the absorption bands become visible in the spectrum.

\section{Comparison to observations}

\subsection{Approximation of Earthshine spectra by weighted sum}

First we approximated the Earthshine observations by the weighted sum method using the fractional surface and cloud cover that have been derived by Sterzik et al. (2012). The fractions in percent are given in the upper part of Table 2. The
Table 2. Top part: contributions of different types of surface to the Earthshine and cloud fractions derived from MODIS data (see Sterzik et al. 2012, Table 1). Bottom part: the corresponding model parameters that were used for the simulations shown in Fig. 8.

\begin{tabular}{lcc}
\hline \hline & \multicolumn{2}{c}{ Observation date } \\
Type & 25 April 2011 & 10 June 2011 \\
\hline Ocean & 18 & 46 \\
Vegetation & 7 & 3 \\
Tundra, shrub, ice, desert & 3 & 1 \\
Total cloud fraction & 72 & 50 \\
Cloud fraction $\tau>6$ & 42 & 27 \\
\hline Model parameters & & \\
\hline Ocean BPDF & 18 & 46 \\
Spectral albedo of grass & 7 & 3 \\
Albedo 0.4 & 3 & 1 \\
Liquid clouds & 42 & 27 \\
Ice clouds & 30 & 23 \\
\hline
\end{tabular}

Notes. All fractions are given in percent.

corresponding model parameters are given in the lower part of the table. We modeled ocean reflection via the BPDF by Mishchenko \& Travis (1997) with a realistic wind speed of $10 \mathrm{~m} \mathrm{~s}^{-1}$ (compare Bentamy et al. 2003, Fig. 7). We tested the effect of wind speed on the polarization spectra and found that it is very small when the full sunglint is in the observation. The width of the sun-glint area increases with increasing wind speed but the integral over it remains approximately the same for all Stokes components. The difference between wind speeds of $1 \mathrm{~m} \mathrm{~s}^{-1}$ and $10 \mathrm{~m} \mathrm{~s}^{-1}$ is less than $0.5 \%$ in $P$. For vegetation we used the spectral albedo of grass as measured by Feister \& Grewe (1995). Other surface types have a very small impact as their maximum fractional contribution is only $3 \%$ at maximum. We used a Lambertian albedo of 0.4 as typical for desert to simulate these other surfaces. The fractional cloud cover and cloud optical thicknesses were derived from MODIS observations. We make the simplified assumption that the optically thicker clouds $(\tau>6)$ are liquid water clouds and the thinner clouds $(\tau<6)$ are ice clouds. We further assume that the liquid water clouds extend in a layer from $2-3 \mathrm{~km}$, have an optical thickness of 10 , and an effective radius of $10 \mu \mathrm{m}$. The ice clouds extend from 10-11 km altitude, have an optical thickness of 1 and an effective radius of $30 \mu \mathrm{m}$. In all simulations we used the standard atmosphere for midlatitude summer (Anderson et al. 1986). The phase angle was set to $90^{\circ}$. We obtain the degree of polarization for the "quasi" inhomogeneous planet according to Eq. (2).

Figure 8 shows simulated (blue) versus observed (gray) spectra of the degree of polarization. The gray area shows the uncertainty of the observed degree of polarization due to the uncertain depolarization of the moon surface (see Sect. 3). The green lines show the best fit that could be obtained using the dataset by Stam (2008), which includes various surface types and liquid water clouds, but no ice water clouds. Further the fit does not include a realistic BPDF to simulate polarized reflection at the ocean surface. Both scattering by high ice water clouds and reflection at water surfaces produce a higher degree of polarization in the red part of the spectrum and decrease the spectral slope, therefore we obtain a much better match with the observation.

Indeed, the simulations for 25 April fit the observations very well within the quite large uncertainty due to the uncertain moon depolarization. The magnitude and slope of the spectrum of the degree of polarization are very similar. The simulations 
C. Emde et al.: Influence of aerosols, clouds, and sunglint on polarization spectra of Earthshine
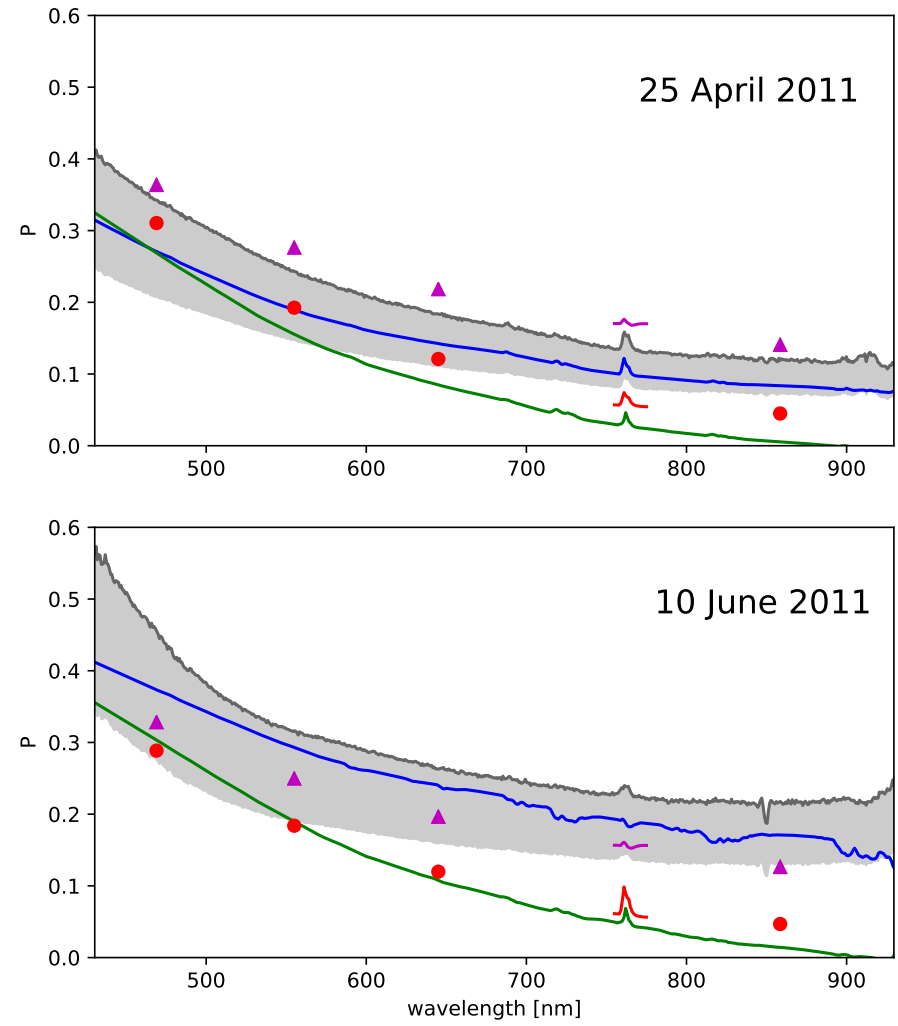

Fig. 8. Observations by FORS against MYSTIC simulations (see text for details). The blue lines correspond to simulations for homogeneous planets, which are averaged using a weighted sum approach (see Sect. 6.1). The red circles correspond to simulations for a realistic Earth model including 3D cloud fields and a 2D surface albedo map derived from MODIS data (see Sect. 6.2.1). The purple triangles indicate a planet with the same 3D cloud fields, but the surface is completely covered with ocean and simulated using a BPDF (see Sect. 6.2.2). The gray area shows the uncertainty range due to the unknown moon depolarization. The green line shows the best fit, which was obtained using the dataset by Stam (2008) without ice clouds (see Sterzik et al. 2012). The top panel represents the observation on 25 April 2011 and the bottom panel 10 June 2011.

for 10 June are also within the uncertainty range of the observation. However, the spectral slope is different. The very steep decrease from $400-500 \mathrm{~nm}$ followed by the rather flat spectrum from 500-900 nm could not be simulated and the increase of the degree of polarization above $900 \mathrm{~nm}$ cannot be reproduced by the model. Further, in the $\mathrm{O}_{2} \mathrm{~A}$ band region, the degree of polarization is decreased compared to the continuum in the model whereas it is increased in the observation. The decrease can be explained by the very high ocean fraction that is modeled using the BPDF. Whereas Lambertian surface reflection is unpolarized, the ocean reflection is highly polarized in the sunglint region. For Lambertian reflection $Q$ and $U$ are zero for radiation that is reflected, hence only $I$ is reduced by absorption so that the degree of polarization is higher in the $\mathrm{O}_{2} \mathrm{~A}$ band than in the continuum. Radiation reflected at the ocean is polarized, hence $I, Q$ and $U$ are decreased by absorption and depending on the reflectance function, the polarization in the $\mathrm{O}_{2} \mathrm{~A}$ band can be higher or lower compared to the continuum. Unpublished Earthshine spectra of the $\mathrm{O}_{2} \mathrm{~A}$ band also exhibit large variability, including the possibility of a smaller band polarization compared to the continuum (Sterzik et al. 2017). Our result shows that the weighted sum approach is not accurate enough to explain the shape of the $\mathrm{O}_{2} \mathrm{~A}$ band.
Table 3. Positions of sun and moon used for the simulations.

\begin{tabular}{lll}
\hline \hline & 25 April 2011, 9:00 UTC & 10 June 2011, 1:00 UTC \\
\hline Sun & $13^{\circ} \mathrm{N}, 45^{\circ} \mathrm{E}$ & $23^{\circ} \mathrm{N}, 165^{\circ} \mathrm{E}$ \\
Moon & $15^{\circ} \mathrm{S}, 39^{\circ} \mathrm{W}$ & $5^{\circ} \mathrm{S}, 95^{\circ} \mathrm{W}$ \\
\hline
\end{tabular}

We know from the results by Karalidi \& Stam (2012) that the approximation by a weighted sum of results for homogeneous planets is not accurate. However, the results shown in this section already indicate that the observations are realistic, in particular we now understand the high degree of polarization in the continuum of the red part of the spectrum.

\subsection{Realistic simulation with $3 D$ cloud fields}

\subsubsection{Simulation with 2D Lambertian surface}

We simulated the Earthshine spectra for a realistic Earth atmosphere including 3D clouds from the ECMWF model as in Sect. 2.2. We used cloud data from the operational forecast system IFS. For the observation on 25 April 2011, 9:00 UTC, we took the $9 \mathrm{~h}$ forecast from midnight (25 April 2011, 0:00 UTC) and for the observation on 10 June, 1:00 UTC, we take the $12 \mathrm{~h}$ forecast from 9 June 2011, 12:00 UTC. As before we used constant effective radii of $10 \mu \mathrm{m}$ for liquid water clouds and $30 \mu \mathrm{m}$ for ice water clouds. Further we multiplied the liquid/ice water contents with the sub-grid cloud cover to obtain correct water contents for each grid cell.

To define the geometrical setup for the MYSTIC simulation, we provide the exact position of the moon with respect to the Earth (latitude and longitude, distance) and the position of the sun. Table 3 shows the positions that were used for the simulations, these setups correspond to the configurations on 25 April 2011 and on 10 June 2011, when Earthshine spectra were observed. Further we define the field of view of the sensor so that it includes the full Earth and set the spatial resolution to $500 \times 500$ pixels.

We used the 2D surface albedo map derived from MODIS data (see Sect. 2.2). The surface is thus treated as Lambertian reflector with different albedos, where the albedo of the ocean is very small. Ocean reflection is not modeled using the BPDF in this section. The molecular atmosphere (US standard) is constant over the full planet and molecular absorption is calculated with REPTRAN.

As mentioned before, we cannot include spectrally dependent cloud optical properties when the ALIS method is used. Therefore we performed only monochromatic calculations for center wavelengths of MODIS channels (469 nm, $555 \mathrm{~nm}$, $645 \mathrm{~nm}$, and $858.5 \mathrm{~nm}$ ), where 2D surface albedo data derived from MODIS are available.

Figure 9 shows the results for all simulated wavelengths. The left images correspond to the intensity (Stokes component $I$ normalized to incoming solar irradiance). At $469 \mathrm{~nm}$ we hardly see the surface because land surfaces reflect only little radiation at short wavelengths and the ocean albedo is close to 0 . The surface looks brightest at $858.5 \mathrm{~nm}$ because at this wavelength the land surface albedo is very high, whereas the clear-sky parts appear very dark because there is only very little Rayleigh scattering. In all images of $Q_{i}$ and $U_{i}$ the land surface is not visible because we assume a Lambertian non-polarizing surface. At the shorter wavelengths $469 \mathrm{~nm}, 555 \mathrm{~nm}$, and $645 \mathrm{~nm}$ the clouds depolarize, i.e., $Q_{i}$ and $U_{i}$ have smaller absolute values above clouds. The depolarization by clouds can also be seen in the 

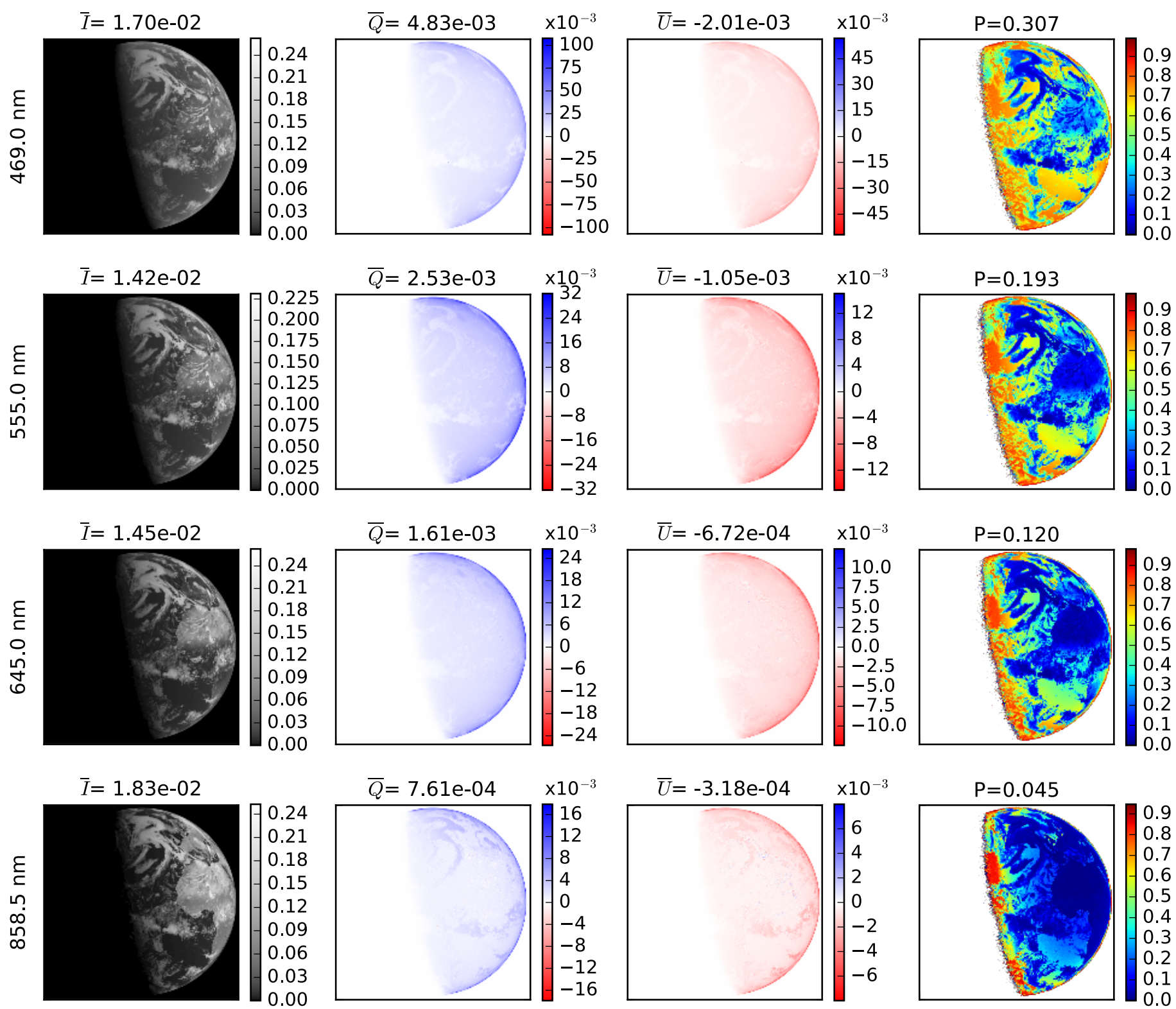

Fig. 9. Simulations of the Earth as seen by the moon on 25 April 2011 at 9:00 UTC. The cloud fields (liquid and ice clouds) are taken from the ECMWF IFS weather forecast model. The surface is included as a $2 \mathrm{D}$ albedo map derived from MODIS observations. The rows correspond to the central wavelengths of MODIS channels. The left plots show the radiance $I$, the middle plots show the linear polarization components $Q$ and $U$, and the right plot shows the degree of polarization $P$. The numbers on top of the figures indicate the averages for $I, Q$, and $U$ and the degree of polarization of the complete image.

image of the degree of polarization $P_{i}$. For $858.5 \mathrm{~nm}$ clouds polarize more strongly than the Rayleigh background, thus they appear darker in the $Q_{i}$ and $U_{i}$ images. The degree of polarization $P_{i}$ is generally very small and close to 0 above the bright land surface. The numbers above the images are mean values of the Stokes vector components $\bar{I}=\sum I_{i} / N, \bar{Q}=\sum Q_{i} / N$ and $\bar{U}=\sum U_{i} / N$, where $N=500^{2}$ is the number of pixels. The degree of polarization $P=\sqrt{\bar{Q}^{2}+\bar{U}^{2}} / \bar{I}$ is written above the image of $P_{i}$. The value $P$ decreases from about 0.3 at $469 \mathrm{~nm}$ to 0.045 at $858.5 \mathrm{~nm}$. These results are indicated as red circles in Fig. 8. The value at $858.5 \mathrm{~nm}$ is clearly lower than the measurement; all other results are within the gray area and hence match the observation within the range of uncertainty. Using the same model setup we also calculate the region about the $\mathrm{O}_{2} \mathrm{~A}$ band from $755 \mathrm{~nm}$ to $775 \mathrm{~nm}$ using ALIS with constant scattering coefficients over the spectral range. We find a relatively strong $\mathrm{O}_{2} \mathrm{~A}$-band polarization compared to the continuum, i.e., stronger than in the observation.

The same simulations were performed for 10 June 2011 and the resulting values of the degree of polarization are also included in Fig. 8. For this observation day the results are far below the observation for $645 \mathrm{~nm}$ and $858.5 \mathrm{~nm}$. The degree of polarization is very similar to the results for the April observation, which means that the globally averaged cloud distributions from the ECMWF model are similar for the two observation times in April and June. In particular the clouds do not explain the observed difference in degree of polarization of almost $10 \%$ in the red part of the spectrum.

Figure 10 shows a true color composite, where red corresponds to $645 \mathrm{~nm}$, green to $555 \mathrm{~nm}$, and blue to $469 \mathrm{~nm}$. The results look very similar to satellite images of the Earth, and indeed 

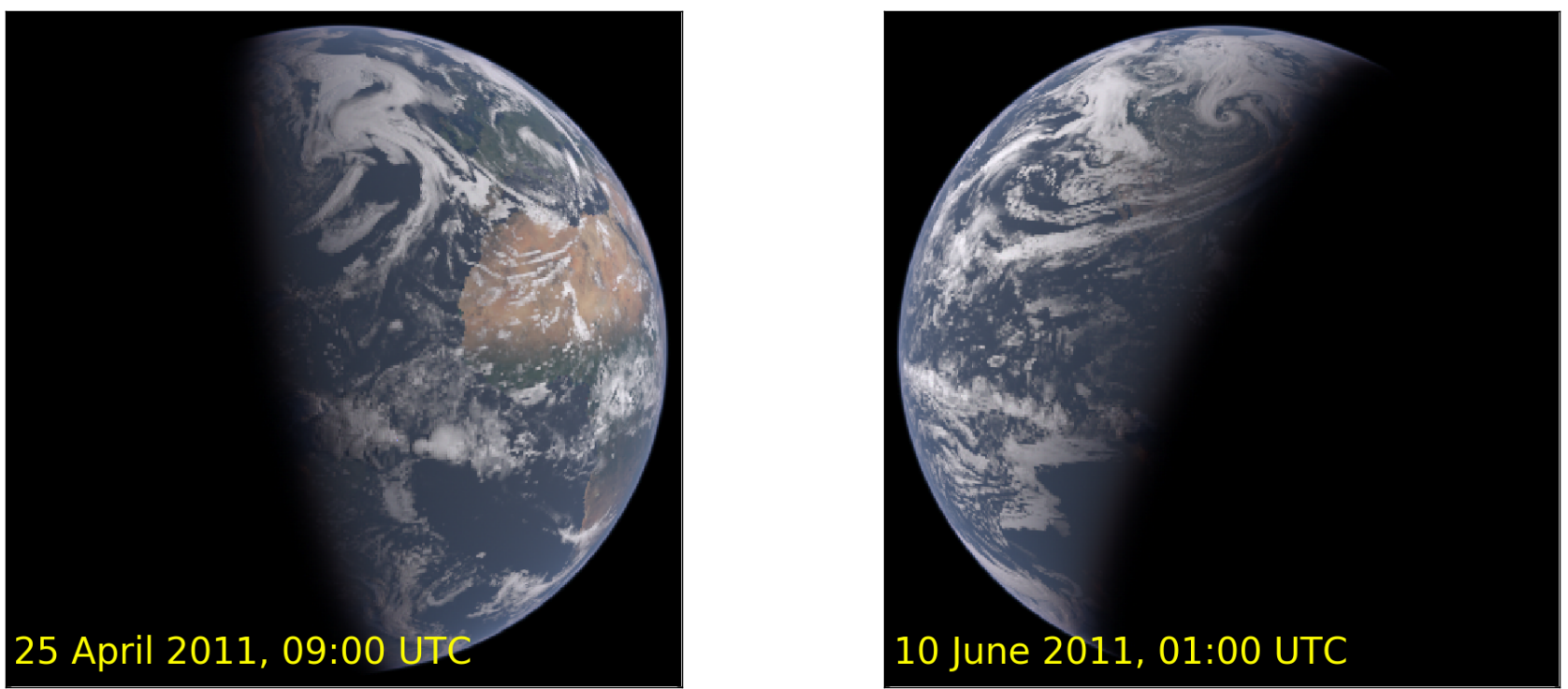

Fig. 10. Simulations of the Earth as seen by the moon. The figure shows a true color composite; red corresponds to $645 \mathrm{~nm}$, green to $555 \mathrm{~nm}$, and blue to $469 \mathrm{~nm}$.

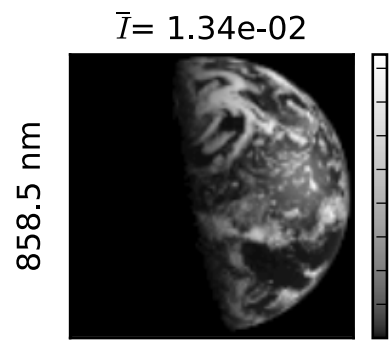

$\bar{I}=1.32 \mathrm{e}-02$

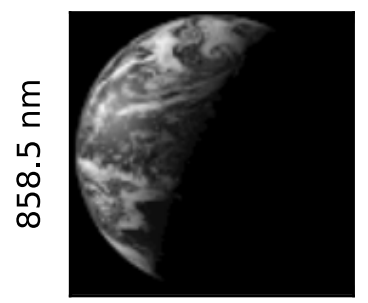

$\bar{Q}=1.74 \mathrm{e}-03$

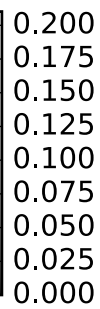

0.000

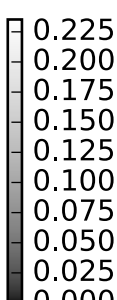

$\bar{Q}=1.17 \mathrm{e}-03$
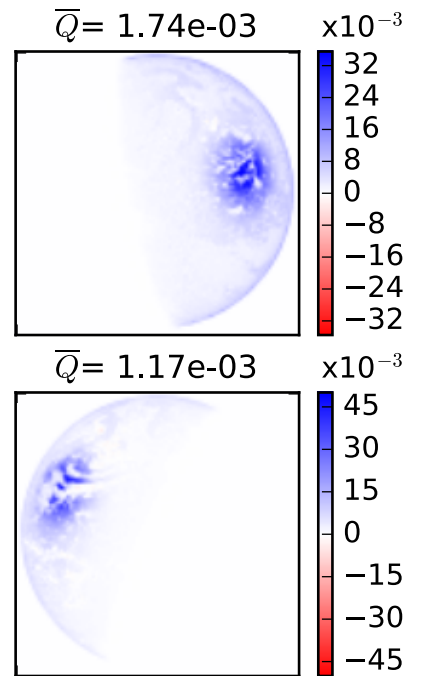

$\times 10^{-3}$

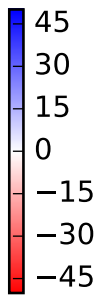

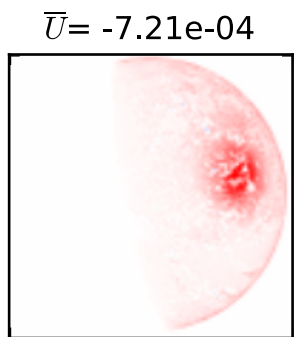
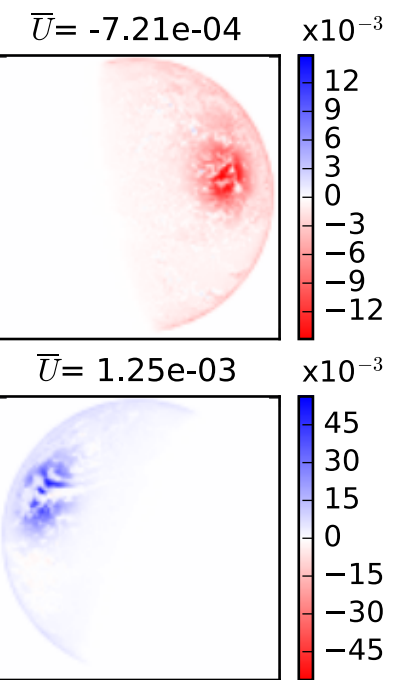

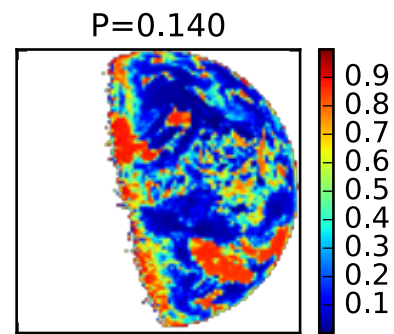

$\mathrm{P}=0.130$

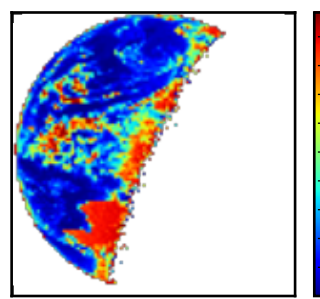

Fig. 11. Simulations for a planet covered with liquid water. The geometrical setup corresponds to the two measurement dates, 25 April 2011 (top) and 10 June 2011 (bottom). The ocean surface reflection is considered by a realistic bi-directional polarized distribution function (BPDF) (Mishchenko \& Travis 1997). The sunglint is clearly visible in the images of the Stokes components $I_{i}, Q_{i}$ and $U_{i}$.

clouds and surface look very realistic. But quantitatively the degree of polarization is too low, especially in the red part of the spectrum for 10 June 2011.

One important aspect is still missing in these simulations: the contribution to the observed polarization due to the scattering of the solar radiation by the surface. Whether or not this explains the remaining differences between simulation and observation is investigated in the next section.

\subsubsection{Polarization by ocean}

Currently our model does not allow us to combine the ocean BPDF with a Lambertian land surface. In order to test the impact of polarization by reflection at the water surface, we performed simulations for a planet completely covered with water. We used a realistic ocean BPDF (Mishchenko \& Travis 1997), which includes also shadowing effects by ocean waves. We assumed a constant wind speed of $10 \mathrm{~m} \mathrm{~s}^{-1}$ and performed monochromatic calculations at $469 \mathrm{~nm}, 555 \mathrm{~nm}, 645 \mathrm{~nm}$, and $858.5 \mathrm{~nm}$. The spatial resolution of the images is set to $100 \times 100$ pixels.

Figure 11 shows the results at $858.5 \mathrm{~nm}$. The top panels are for 25 April 2011 and the bottom panels for 10 June 2011. The images of $I_{i}$ show the sunglint as a bright area. In the images of $Q_{i}$ and $U_{i}$, the high polarization in the sunglint region is clearly seen. The pixel-average $\bar{Q}$ and $\bar{U}$ are significantly higher than in the simulations for the 2D-albedo map from MODIS data, and this yields an increase of about $10 \%$ in degree of polarization $P$. When we compare Figs. 10 and 11 we see that the sunglint on 25 April 2011 at 9:00 UTC would be located on the Sahara desert, thus at this time we do not expect much polarization from radiation reflected by the ocean. On 10 June at 1:00 UTC the sunglint is located in the middle of the Pacific ocean, so we can expect to see it clearly in the observation. The results of all simulations for a completely water covered planet are included as magenta triangles in Fig. 8.

On 25 April the predicted degree of polarization for the planet completely covered with water is higher than the 
Table 4. Computational time on one CPU (Intel(R) Xeon(R) CPU E5$2630 \mathrm{v} 4 @ 2.20 \mathrm{GHz})$ for the full spectra (1000 wavelengths) of the degree of polarization and corresponding spectrally averaged relative standard deviations $\sigma$.

\begin{tabular}{lccc}
\hline \hline Homogeneous planets & $\bar{P}$ & $\overline{\sigma(P) / P}$ & Time [s] \\
\hline BPDF (Tsang) & 0.84 & 0.010 & 477 \\
Albedo 0.4 & 0.18 & 0.015 & 490 \\
Spectral albedo of grass & 0.46 & 0.010 & 480 \\
Liquid cloud $(\tau=10.0)$ & 0.06 & 0.088 & 802 \\
Ice cloud $(\tau=1.0)$ & 0.21 & 0.025 & 687 \\
\hline Inhomogeneous planets & $P$ & $\sigma(P) / P$ & time [s] \\
\hline ECMWF clouds \& MODIS albedo & 0.18 & 0.006 & 29 \\
ECMWF clouds \& BPDF & 0.25 & 0.005 & 28 \\
\hline
\end{tabular}

Notes. The simulations were performed with $N_{\mathrm{ph}}=10^{5}$ photons. The computational time is proportional to $N_{\text {ph }}$ and the corresponding standard deviation $\sigma$ is proportional to $N_{\mathrm{ph}}^{-1 / 2}$. The numbers for the scenarios with ECMWF clouds correspond to monochromatic calculations at $555 \mathrm{~nm}$ for 10 June 2011.

observation (purple triangles). For this day the simulation with the 2D Lambertian surface albedo (red circles) is more realistic than the simulation for a water covered planet. The remaining difference between observation and simulation (red circles) can be explained by the completely missing polarization contribution by the surface in the simulation with Lambertian surface.

On 10 June the simulations for the water covered planet (magenta triangles) are more realistic and indeed they lie in the gray area corresponding to the uncertainty range of the observation.

The results clearly show that the difference between the two observed spectra of about $10 \%$ in the red part of the spectrum is due to the polarized reflection of the ocean surface, in particular in the sunglint region.

\subsection{Computational times}

The computational times for the cases shown in Fig. 8 are summarized in Table 4. In the Monte Carlo codes the computational time is proportional to the number of photons $N_{\mathrm{ph}}$ used for the simulation and the standard deviation is proportional to $N_{\mathrm{ph}}^{-1 / 2}$. All results shown in the table are for $N_{\mathrm{ph}}=10^{5}$. For the homogeneous planet simulations we used the absorption lines importance sampling method, ALIS, and the given times are for full spectra with 1000 grid points. The clear-sky simulations take less than $10 \mathrm{~min}$ and simulations with clouds take less than $15 \mathrm{~min}$. For the inhomogeneous planets including the 3D cloud fields the computational time corresponds to monochromatic calculations. These simulations (with 2D albedo map or BPDF) take about $0.5 \mathrm{~min}$. The images require significantly more computational time. If we want to have the same accuracy for each pixel we have to multiply the given computational time by the number of pixels; for example, for the images with $100 \times 100$ pixels the computational time is increased by a factor of $10^{4}$.

\section{Conclusions}

We have presented a novel approach to simulate the polarized radiation scattered by Earth as seen from space, which is of general significance also for future polarimetric observations of Earth-like exoplanets. The approach has been implemented in the Monte Carlo code MYSTIC, which allows us to compute the intensity and degree of polarization with high accuracy and high spectral resolution. We validated the outputs of the code by comparing its predictions with the data tabulated by Stam (2008) for homogeneous planets with different surfaces, and we consistently found very good agreement. Then we investigated the impact of various atmospheric components: aerosols, water clouds, and ice clouds, as well as that of various kinds of surface. We found, for instance, that the presence of high ice clouds in the atmosphere increases the degree of polarization in the red part of the spectrum and that the polarization due to the scattering of the light from the ocean has a large impact if the sunglint over ocean is visible to the observer. The potential to detect enhanced reflectivity from an ocean glint and its effect on polarization in the phase curve of an Earth-like exoplanet has been previously advocated by Williams \& Gaidos (2008).

We used our code to try to interpret the Earthshine optical spectropolarimetric observations obtained in April and June 2011 with the FORS instrument of the ESO VLT by Sterzik et al. (2012). In particular we were interested in explaining the high value of the polarization observed at longer wavelengths that could not be interpreted in the previous modeling attempts. We performed simulations considering realistic 3D fields of liquid water and ice water clouds, which we obtained from the ECMWF operational weather forecast model at the times of the FORS observations, and 2D albedo maps of the planet surface, which were derived from MODIS observations. This way we obtained very similar results for the two specific observing epochs, fitting the April data well, but not the observations obtained in June, which exhibited a degree of polarization almost $10 \%$ higher in the red part of the spectrum than those obtained in April. We then performed a simulation with the same cloud fields, but for a planet surface completely covered by water, using a realistic bi-directional polarized distribution function. By so doing, we substantially improved the fit to the observations obtained in June. By simulating images of the Earth as seen by the Moon we found that in June, the position of the sunglint is above the Pacific ocean, whereas in April the position would be above the Sahara, and thus the observation in April does not include the sunglint. Our modeling results show that the degree of polarization in the red part of the spectrum increases by about $10 \%$ when the sunglint is observed; this increase corresponds to the observed difference between the two observation epochs.

In conclusion, compared to previous modeling results, the inclusion of ice clouds allowed us a substantial improvement of the fit to the data obtained in both observing epochs. We then showed that the enhanced polarization fraction caused by the sunglint over ocean can be clearly identified in the red part of the Earthshine spectra obtained in June. This finding may be of significance in the interpretation of future polarimetric measurements of exosolar planets.

To further improve the modeling of the polarization properties of the radiation scattered by Earth, the following steps are needed:

\footnotetext{
- Allowing the simultaneous inclusion of BPDF and Lambertian surfaces.

- Implementation of BPDFs for land surfaces.

- Inclusion of 3D aerosol data.
}

A major difficulty in the interpretation of the Earthshine observations is the uncertain contribution of the depolarization of the moon surface. In that respect, a fully reliable modeling of the light scattered by Earth could be actually used to measure the depolarization matrix of the moon surface from Earthshine measurements. 
Future applications of our model may include the interpretation of spectropolarimetric observations in the near-infrared spectral range (e.g., Miles-Paéz et al. 2014) and the simulation of images of the degree of polarization of other planets of the solar system, for example, using the Jupiter maps observed by McLean et al. (2017).

Acknowledgements. Based on observations made with ESO Telescopes at the La Silla Paranal Observatory under program 87.C-0040. We thank the anonymous reviewer for helpful comments and suggestions that helped us to improve our manuscript.

\section{References}

Anderson, G., Clough, S., Kneizys, F., Chetwynd, J., \& Shettle, E. 1986, AFGL atmospheric constituent profiles (0-120 km), Tech. Rep. AFGL-TR-86-0110, Air Force Geophys. Lab., Hanscom Air Force Base, Bedford, Mass.

Baum, B. A., Yang, P., Heymsfield, A. J., et al. 2014, J. Quant. Spectr. Rad. Transf., special Issue ELS-XIV

Bazzon, A., Schmid, H. M., \& Gisler, D. 2013, A\&A, 556, A117

Bentamy, A., Katsaros, K. B., Nuñez, A. M. M.-N., et al. 2003, J. Clim., 16, 637

Boesche, E., Stammes, P., Preusker, R., et al. 2008, Appl. Opt., 47, 3467

Buras, R., \& Mayer, B. 2011, J. Quant. Spectr. Rad. Transf., 112, 434

Cox, C., \& Munk, W. 1954a, J. Opt. Soc. Am., 44, 838

Cox, C., \& Munk, W. 1954b, J. Mar. Res., 13, 198

Davis, A. B., Garay, M. J., Xu, F., Qu, Z., \& Emde, C. 2013, Proc. SPIE, 8873, 887309

de Haan, J. F., Bosma, P. B., \& Hovenier, J. W. 1987, A\&A, 183, 371

Deutschmann, T., Beirle, S., Frieß, U., et al. 2011, J. Quant. Spectr. Rad. Trans., 112,1119

Dollfus, A. 1957, Suppléments aux Annales d'Astrophysique, 4, 3

Emde, C., \& Mayer, B. 2007, Atm. Chem. Phys., 7, 2259

Emde, C., Buras, R., Mayer, B., \& Blumthaler, M. 2010, Atm. Chem. Phys., 10, 383

Emde, C., Buras, R., \& Mayer, B. 2011, J. Quant. Spectr. Rad. Transf., 112, 1622

Emde, C., Barlakas, V., Cornet, C., et al. 2015, J. Quant. Spectr. Rad. Transf., 164,8

Emde, C., Buras-Schnell, R., Kylling, A., et al. 2016, Geophys. Mod. Dev., 9, 1647

Eriksson, P., Buehler, S. A., Davis, C. P., Emde, C., \& Lemke, O. 2011, J. Quant. Spectr. Rad. Transf., 112, 1551

Feister, U., \& Grewe, R. 1995, Photochem. Photobiol., 62, 736

García Muñoz, A. 2015, Int. J. Astrobiol., 14, 379
García Muñoz, A., \& Mills, F. P. 2015, A\&A, 573, A72

Gasteiger, J., Emde, C., Mayer, B., et al. 2014, J. Quant. Spectr. Rad. Transf., 148,99

Hapke, B. W., Nelson, R. M., \& Smythe, W. D. 1993, Science, 260, 509

Hess, M., Koepke, P., \& Schult, I. 1998, Bull. Am. Meteor. Soc., 79, 831

Heymsfield, A. J., Schmitt, C., \& Bansemer, A. 2013, J. Atm. Sci., 70, 4123

Karalidi, T., \& Stam, D. M. 2012, A\&A, 546, A56

Karalidi, T., Stam, D. M., \& Hovenier, J. W. 2012, A\&A, 548, A90

Kokhanovsky, A. A., Budak, V. P., Cornet, C., et al. 2010, J. Quant. Spectr. Rad. Transf., 111, 1931

Marchuk, G. I., Mikhailov, G. A., \& Nazaraliev, M. A. 1980, The Monte Carlo methods in atmospheric optics, Springer Series in Optical Sciences (Berlin: Springer)

Marshak, A., \& Davis, A. 2005, 3D Radiative Transfer in Cloudy Atmospheres (Springer)

Mayer, B. 2009, EPJ Conf., 1, 75

McClatchey, R., Fenn, R., Selby, J., Volz, F., \& Garing, J. 1972, Optical properties of the Atmosphere, AFCRL-72.0497, Tech. Rep., Us Air Force Cambridge Research Labs

McLean, W., Stam, D., Bagnulo, S., et al. 2017, A\&A, 601, A142

Miles-Paéz, P. A., Pallé, E., \& Osorio, M. R. Z. 2014, A\&A, 562, L5

Mishchenko, M. I., \& Travis, L. D. 1997, J. Geophys. Res., 102, 16989

Schaaf, C. B., Gao, F., Strahler, A. H., et al. 2002, Remote Sensing of Environment, 83, 135, the Moderate Resolution Imaging Spectroradiometer (MODIS): a new generation of Land Surface Monitoring

Schmidt, H., Beuzit, J., Feldt, M., et al. 2006, Direct Imaging of Exoplanets: Science \& Techniques (IAU Coll. 200), 165

Seager, S., Whitney, B. A., \& Sasselov, D. D. 2000, ApJ, 540, 504

Stam, D. M. 2008, A\&A, 482, 989

Stam, D., Haan, J. D., Hovenier, J., \& Stammes, P. 2000, J. Quant. Spectr. Rad. Transf., 64, 131

Stam, D. M., de Rooij, W. A., Cornet, G., \& Hovenier, J. W. 2006, A\&A, 452, 669

Stap, F., Hasekamp, O., Emde, C., \& Röckmann, T. 2016, J. Quant. Spectr. Rad. Transf., 170, 54

Sterzik, M. F., Bagnulo, S., \& Palle, E. 2012, Nature, 483, 64

Sterzik, M., Bagnulo, S., Emde, C., \& Stam, D. 2017, Contribution to the ESO calibration workshop, January 16-19, Santiago de Chile

Stevenson, K. B., Harrington, J., Nymeyer, S., et al. 2010, Nature, 464, 1161

Takahashi, J., Itoh, Y., Akitaya, H., et al. 2013, PASJ, 65, 38

Tsang, L., Kong, J. A., \& Shin, R. T. 1985, Theory of Microwave Remote Sensing (New York: John Wiley), 613

Wang, C., Yang, P., Baum, B. A., et al. 2011, J. Appl. Meteor. Clim., 50, 2283

Williams, D. M., \& Gaidos, E. 2008, Icarus, 195, 927

Wiscombe, W. 1980, Appl. Opt., 19, 1505

Yang, P., Bi, L., Baum, B. A., et al. 2013, J. Atm. Sci., 70, 330 